\title{
A GENERALIZED STRANGE TERM IN SIGNORINI'S TYPE PROBLEMS
}

\author{
${\text { Carlos } \text { Conca }^{1}, \text { François Murat }^{2} \text { and Claudia Timofte }}^{3}$
}

\begin{abstract}
The limit behavior of the solutions of Signorini's type-like problems in periodically perforated domains with period $\varepsilon$ is studied. The main feature of this limit behaviour is the existence of a critical size of the perforations that separates different emerging phenomena as $\varepsilon \rightarrow 0$. In the critical case, it is shown that Signorini's problem converges to a problem associated to a new operator which is the sum of a standard homogenized operator and an extra zero order term ("strange term") coming from the geometry; its appearance is due to the special size of the holes. The limit problem captures the two sources of oscillations involved in this kind of free boundary-value problems, namely, those arising from the size of the holes and those due to the periodic inhomogeneity of the medium. The main ingredient of the method used in the proof is an explicit construction of suitable test functions which provide a good understanding of the interactions between the above mentioned sources of oscillations.
\end{abstract}

Mathematics Subject Classification. 35B27, 35A25, 42C30.

Received: February 17, 2003.

\section{INTRODUCTION}

The general question which will make the object of this paper is the homogenization of Signorini's type-like problems in perforated domains. Their classical weak formulations involve a standard variational inequality (in the sense of Lions and Stampacchia [22]), corresponding to a nonlinear free boundary-value problem. Such a model was introduced in the earliest '30 by Signorini [25] (see also Fichera [20]) for studying problems arising in elasticity, and more precisely problems involving an elastic body under unilateral contact shear forces acting on its boundary. For a nice presentation of the mechanical aspects behind Signorini's problem (and also for some mathematical and historical backgrounds) we refer to Duvaut and Lions [19] (see also Brézis [4]).

Let $\Omega$ be an open bounded set in $\mathbb{R}^{n}$ and let us perforate it by holes. As a result, we obtain an open set $\Omega^{\varepsilon}$ which will be referred to as being the perforated domain; $\varepsilon$ represents a small parameter related to the characteristic size of the perforations. As usual in homogenization, we shall be interested in obtaining a suitable description of the asymptotic behavior, as $\varepsilon$ tends to zero, of the solution $u^{\varepsilon}$ in such domains. We will wonder, for example, whether the solution $u^{\varepsilon}$ converges to a limit $u$ as $\varepsilon \rightarrow 0$. And if this limit exists, can it be characterized?

\footnotetext{
Keywords and phrases. Signorini's problem, homogenization, Tartar's method, variational inequality.

1 Departamento de Ingeniería Matemática and Centro de Modelamiento Matemático, UMR 2071 CNRS-UChile, Facultad de Ciencias Físicas y Matemáticas, Universidad de Chile, Casilla 170/3, Santiago, Chile. e-mail: cconca@dim.uchile.cl

2 Laboratoire Jacques-Louis Lions, Université Paris VI, Boîte courrier 187, 75252 Paris Cedex 05, France. e-mail: murat@ann.jussieu.fr

3 Department of Mathematics, Faculty of Physics, University of Bucharest, PO Box MG-11, Bucharest-Magurele, Romania. e-mail: claudiatimofte@hotmail.com 
Let us consider a family of inhomogeneous media occupying the region $\Omega$, parameterized by $\varepsilon$ and represented by $n \times n$ coercive matrices $A^{\varepsilon}(x)$ with real-valued entries defined on $\Omega$. The positive parameter $\varepsilon$ will thus also define a length scale measuring how densely the inhomogeneities of the medium are distributed in $\Omega$. Indeed, one of our main goals in this paper will be to understand the interactions between these two sources of oscillations represented by the parameter $\varepsilon$, namely, those coming from the geometry (more exactly, the size of the holes) and those due to the inhomogeneity of the medium (the rapidly oscillating entries of the matrix $A^{\varepsilon}(x)$ ).

With $\Omega^{\varepsilon}$ we associate the following nonempty closed convex subset of $H^{1}\left(\Omega^{\varepsilon}\right)$ :

$$
K^{\varepsilon}=\left\{v \in H^{1}\left(\Omega^{\varepsilon}\right) \mid v=0 \text { on } \partial \Omega, v \geq 0 \text { on } S^{\varepsilon}\right\}
$$

where $S^{\varepsilon}$ is the boundary of the holes and $\partial \Omega$ is the external boundary of $\Omega$ (we assume that $S^{\varepsilon} \cup \partial \Omega=\emptyset$ ). Our main motivation is to study the asymptotic behavior of the solution of the following variational problem in $\Omega^{\varepsilon}$ :

$$
\left\{\begin{array}{l}
\text { Find } u^{\varepsilon} \in K^{\varepsilon} \text { such that } \\
\int_{\Omega^{\varepsilon}} A^{\varepsilon} D u^{\varepsilon} D\left(v^{\varepsilon}-u^{\varepsilon}\right) \mathrm{d} x \geq \int_{\Omega^{\varepsilon}} f\left(v^{\varepsilon}-u^{\varepsilon}\right) \mathrm{d} x \quad \forall v^{\varepsilon} \in K^{\varepsilon},
\end{array}\right.
$$

where $f$ is a given function in $L^{2}(\Omega)$.

The solution $u^{\varepsilon}$ of (1.2) is also known to be characterized as being the solution of the following non-linear free boundary-value problem: Find a function $u^{\varepsilon}$ and two subsets $S_{0}^{\varepsilon}$ and $S_{+}^{\varepsilon}$ such that $S_{0}^{\varepsilon} \cup S_{+}^{\varepsilon}=S^{\varepsilon}, S_{0}^{\varepsilon} \cap S_{+}^{\varepsilon}=\emptyset$, and

$$
\left\{\begin{array}{l}
-\operatorname{div}\left(A^{\varepsilon} D u^{\varepsilon}\right)=f \text { in } \Omega^{\varepsilon} \\
u^{\varepsilon}=0 \text { on } S_{0}^{\varepsilon}, A^{\varepsilon} D u^{\varepsilon} \cdot \nu \geq 0 \text { on } S_{0}^{\varepsilon}, \\
u^{\varepsilon}>0 \text { on } S_{+}^{\varepsilon}, A^{\varepsilon} D u^{\varepsilon} \cdot \nu=0 \text { on } S_{+}^{\varepsilon},
\end{array}\right.
$$

where $\nu$ is the exterior unit normal to the surface $S^{\varepsilon}$. This means that we can distinguish on $S^{\varepsilon}$ two a priori unknown subsets $S_{0}^{\varepsilon}$ and $S_{+}^{\varepsilon}$ where $u^{\varepsilon}$ satisfies complementary boundary conditions coming from the following global constraints:

$$
u^{\varepsilon} \geq 0, A^{\varepsilon} D u^{\varepsilon} \cdot \nu \geq 0 \text { and } u^{\varepsilon} A^{\varepsilon} D u^{\varepsilon} \cdot \nu=0 \text { on } S^{\varepsilon} .
$$

In this paper, we will be concerned with a special type of heterogeneities of the medium, called periodic structures which are defined by

$$
A^{\varepsilon}(x)=A\left(\frac{x}{\varepsilon}\right)
$$

Here $A=A(y)$ is a continuous matrix-valued function on $\mathbb{R}^{n}$ which is $Y$-periodic and $Y=\left(-\frac{1}{2}, \frac{1}{2}\right)^{n}$ is the basic cell. From a geometrical point of view, we will just consider periodic perforated structures obtained by removing periodically from $\Omega$, with period $\varepsilon$, an elementary hole $T$ which has been appropriated rescaled. In the sequel, we reserve the symbol \# to denote periodicity properties.

We introduce some non-degeneracy assumptions on the materials to ensure the well-posedness of the above problem (1.2):

$$
\left\{\begin{array}{l}
A \in L_{\#}^{\infty}\left(\mathbb{R}^{n}\right)^{n \times n}, \text { or when necessary } A \in \mathcal{C}_{\#}^{0}\left(\mathbb{R}^{n}\right) \text { or even } A \in W_{\#}^{1, \infty}\left(\mathbb{R}^{n}\right)^{n \times n}, \\
A \text { is a symmetric matrix, } \\
\text { for some } 0<\alpha<\beta, \text { one has } \alpha|\xi|^{2} \leq A(y) \xi \cdot \xi \leq \beta|\xi|^{2} \quad \forall \xi, y \in \mathbb{R}^{n} .
\end{array}\right.
$$

Under the above hypotheses, it is well-known by a classical existence and uniqueness result of Lions and Stampacchia [22] that (1.2) is a well-posed problem.

Several situations can occur depending on the asymptotic behavior of the size of the holes. The main feature of our results shows the existence of a critical size that separates different behaviors of the solution $u^{\varepsilon}$ as $\varepsilon \rightarrow 0$. This size is of order $\varepsilon^{n /(n-2)}$ if $n \geq 3$ and of order $\exp \left(-1 / \varepsilon^{2}\right)$ if $n=2$. To simplify matters, in what follows we shall just focus on the case $n \geq 3$, which will be treated explicitly. The case $n=2$ is completely analogous, but since most of the formulas involved have different analytic expressions, we shall omit to treat it. For details in somewhat similar computations, the interested reader is referred to $[9,10]$. 
Besides this so-called critical case (referred to as Case $\mathbf{1}$ throughout the paper and described in the next paragraph below), there are three other cases that will be also considered explicitly. Case $\mathbf{2}$ is characterized by the fact that the holes are much smaller than the critical ones. As expected, in this case they are too small to produce any visible contribution at the limit. So the solution $u^{\varepsilon}$ will just converge to the solution of a classical homogenized Dirichlet problem in $\Omega$ associated to the matrix $A$. Case $\mathbf{3}$ is when the size of the holes is bigger than the critical one, but still smaller than the period $\varepsilon$. The holes being big enough, the positivity constraint of the solution $u^{\varepsilon}$ imposed only on $S^{\varepsilon}$ will become at the limit the positivity condition, $u \geq 0$, on the whole of the domain $\Omega$. The limit problem will be therefore an obstacle problem associated to the corresponding homogenized medium. Then, in this case, the holes don't play an important role in the homogenized problem, they just spread the positivity condition all over the domain. Case $\mathbf{4}$ is similar to the previous one and it is characterized by the fact that the size of the holes is exactly of order $\varepsilon$. The solution $u^{\varepsilon}$ also converges in this case to an obstacle problem, but associated to the homogenization of a periodic heterogeneous and perforated medium. The influence of the holes comes twofold: on one hand, they spread the positivity condition on $S^{\varepsilon}$ to the whole of $\Omega$ and on the other one, their size do affect the homogenized medium.

In the critical case (Case 1), the limit of $u^{\varepsilon}$ is the solution of a problem in $\Omega$ associated with a new operator which is the sum of the standard homogenized one as in Case $\mathbf{2}$ or 3, and of an extra term ("the strange term") that comes in from the geometry (the size of the holes). Be aware that this extra term only appears in this particular case, showing the importance of their size in front of their shape. More precisely, the solution $u^{\varepsilon}$ converges to the unique solution of the following variational equality:

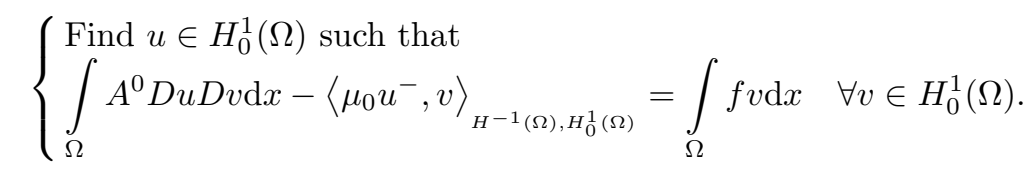

Here, $A^{0}$ is the classical homogenized matrix, whose entries are defined as follows:

$$
a_{i j}^{0}=\frac{1}{|Y|} \int_{Y}\left(a_{i j}(y)+a_{i k}(y) \frac{\partial \chi_{j}}{\partial y_{k}}\right) \mathrm{d} y
$$

in terms of the functions $\chi_{j}, j=1, \ldots, n$, solutions of the so-called cell problems

$$
\left\{\begin{array}{l}
-\operatorname{div}_{y}\left(A(y) D_{y}\left(y_{j}+\chi_{j}\right)\right)=0 \text { in } \mathbb{R}^{n} \\
\chi_{j}-Y \text { periodic }
\end{array}\right.
$$

and $\mu_{0}$ is given by

$$
\mu_{0}=\inf _{\zeta \in H^{1}\left(\mathbb{R}^{n}\right)}\left\{\int_{\mathbb{R}^{n}} A(0) D \zeta D \zeta \mathrm{d} x \mid \zeta \geq 1 \text { q.e. on } T\right\},
$$

$T$ being the elementary hole, and the holes of $\Omega^{\varepsilon}$ being of the size $\varepsilon^{n /(n-2)}$ and periodically distributed with $\operatorname{period} \varepsilon$.

In this limit problem we can see that all the ingredients of our Signorini's inequality (1.2) are present. As already mentioned, two sources of oscillations are involved in (1.2) and we can now see that both are captured at the limit. Those coming from the periodic heterogeneous structure of the medium are reflected by the presence of the homogenized matrix $A^{0}$, and those due to the critical size of the holes are reflected by the appearance of the strange term $\mu_{0}$. The second ingredient contained in (1.5) is the spreading effect of the unilateral condition $u^{\varepsilon} \geq 0$ on $S^{\varepsilon}$ which can be seen by the fact that the strange term only charges the negative part of $u$; it is indeed just the negative part $u^{-}$that is penalized at the limit.

The method we will use is the one introduced by Tartar $[27,28]$ for studying homogenization problems. It consists of constructing suitable test functions that are used in our variational inequalities. 
In studying the general problem (1.2) we will consider three increasingly levels of generality for the matrix $A$. In Section 2 we start by analyzing the simplest case, that of the operator $-\Delta$, which corresponds to $A=I$. Section 3 deals with the so-called regular materials in the sense of [11], materials which are characterized by a divergence-free matrix $A$. The general case is considered in Section 4, by using suitable test functions whose construction is based on a general rearrangement technique due to De Giorgi [18] (see also [1]). This allows us to construct an $a d$-hoc test function which enables us to capture simultaneously both sources of oscillations already mentioned. The first two steps ( $A=I$ and regular materials) do not require such a sophisticated construction of the test functions since in these cases there is no interaction between our two different sources of oscillations; at most one of the source is significant at the limit.

This paper is a generalization of the well-known seminal work of Cioranescu and Murat [9]. In this article, the authors deal with the asymptotic behavior of solutions of Dirichlet problems in perforated domains, showing the appearance of a "strange" extra-term as the period of the perforations tends to zero and the holes are of critical size. They also consider the constraint $u^{\varepsilon} \geq 0$ on the holes. In our paper, we generalize their method and framework to a class of Signorini's problem, involving just a positivity condition imposed on the boundary of the holes. Our results show that one is led to analogous limit problems despite the fact that the constraint is only acting on $S^{\varepsilon}$.

One of the main elements of our method is the existence of some special test functions $w^{\varepsilon}$ previously introduced in the original paper [7] and satisfying hypotheses (H.0)-(H.5) below (see Chap. 2). It is wellknown that these hypotheses force some geometrical restrictions, even if it was proved by J. Casado-Díaz [6] that the existence of functions $w^{\varepsilon}$ vanishing on $T^{\varepsilon}$ and weakly converging to 1 in $H^{1}(\Omega)$ (hypotheses (H.1)(H.3)) is sufficient. Another method was introduced by V.A. Marcenko and E.Ja. Hrouslov in their book [23] and then developed in the setting of nonlinear problems by I.V. Skrypnik [26]. This method also undergoes geometrical restrictions. Recently, G. Dal Maso and A. Garroni [15] were able to overcome this weakness of the method by using different test functions. The introduction of these test functions allowed them to recover by the method of test functions due to Tartar previous results obtained for the Dirichlet problem with holes by the $\Gamma$-convergence method without making any assumption on the geometry (most of these results are due to G. Dal Maso, see e.g. [12], [13]). It also opened new ways for solving more general cases of Dirichlet problems for monotone operators (see G. Dal Maso and F. Murat [16] and J. Casado-Díaz [7]). Also, notice that in a recent paper, G. Dal Maso and F. Murat [17] (see also A. Kovalevsky [21]) are addressing the very general problem of finding the asymptotic behavior of solutions of linear Dirichlet problems with simultaneously varying operators and domains, emphasizing the interest of understanding the interaction between these two sources of oscillations. This result was generalized to the case of Dirichlet problems for monotone operators by C. Calvo-Jurado and J. Casado-Díaz in [5].

As already mentioned, the method we follow in the present paper is the method of Tartar [27]. However, it is worth mentioning that the $\Gamma$-convergence of integral functionals involving oscillating obstacles is an alternative which already proved to be a successful one. Extensive references on this topic can be found in the monographs of Dal Maso [14] and of Braides and Defranceschi [3]. For example, our main result concerning the critical case (it establishes that $u^{\varepsilon}$ converges to the solution of (1.5); see Th. 4.1 below) can also be interpreted as the $\Gamma$-convergence of the functionals

$$
v \longmapsto \int_{\Omega^{\varepsilon}} A\left(\frac{x}{\varepsilon}\right) D v D v \mathrm{~d} x-2 \int_{\Omega^{\varepsilon}} f v \mathrm{~d} x+I_{K^{\varepsilon}}(v)
$$

(where $I_{K^{\varepsilon}}$ is the indicator function of the set $K^{\varepsilon}$, i.e. $I_{K^{\varepsilon}}$ is equal to zero if $v$ belongs to $K^{\varepsilon}$ and $+\infty$ otherwise) to the limit functional

$$
v \longmapsto \int_{\Omega} A^{0} D v D v \mathrm{~d} x-2 \int_{\Omega} f v \mathrm{~d} x+\left\langle\mu_{0},\left(v^{-}\right)^{2}\right\rangle_{H^{-1}(\Omega), H_{0}^{1}(\Omega)},
$$

which is the energy functional associated to (1.5). 
Finally, a word about the notation adopted in this work. Apart from the usual norms in Sobolev spaces $H^{1}(\Omega)$, $H^{2}(\Omega)$, we will use the following semi-norms,

$$
|v|_{H^{1}(\Omega)}^{2}=\sum_{j=1}^{n}\left\|\frac{\partial v}{\partial x_{j}}\right\|_{L^{2}(\Omega)}^{2}, \quad|v|_{H^{2}(\Omega)}^{2}=\sum_{i, j=1}^{n}\left\|\frac{\partial^{2} v}{\partial x_{i} \partial x_{j}}\right\|_{L^{2}(\Omega)}^{2}
$$

Also, throughout the paper, by $C$ we shall denote a generic fixed strictly positive constant, whose value can change from line to line.

\section{The Classical Strange term in Signorini's PROBlem}

In this chapter, we will consider the classical Signorini's problem for the operator $-\Delta$ in periodically perforated domains. We will treat separately the situation in which the size of the holes is strictly smaller than the distance between them and the situation in which the holes are of similar size compared to the period. The first one will be referred to as the case of small holes, while for the second one we shall use the terminology of big holes.

Let $\Omega$ be a smooth bounded connected open subset of $\mathbb{R}^{n}$ and let $T$ be another open bounded subset of $\mathbb{R}^{n}$, with a smooth boundary $\partial T$ (of class $C^{2}$ ). We shall refer to $T$ as being the elementary hole. We assume that 0 belongs to $T$ and that $T$ is star-shaped with respect to 0 . Since $T$ is bounded, to simplify matters, without loss of generality, we shall assume that $\bar{T} \subset Y$, where $Y=\left(-\frac{1}{2}, \frac{1}{2}\right)^{n}$ is the representative cell in $\mathbb{R}^{n}$.

Let $\varepsilon$ be a real parameter taking values in a sequence of positive numbers converging to zero and let $r: \mathbb{R}_{+} \rightarrow$ $\mathbb{R}_{+}$be a continuous map, which will allow us to distinguish between the above mentioned situations. In the sequel, we will make various further assumptions on the asymptotic behavior of this function, as $\varepsilon \rightarrow 0$. Some of them are as follows:

$$
\lim _{\varepsilon \rightarrow 0} \frac{r(\varepsilon)}{\varepsilon}=0 \text { and } r(\varepsilon)<\varepsilon / 2
$$

and

$$
r(\varepsilon) \sim \varepsilon,
$$

this last one meaning, for example, that $r(\varepsilon)=C \varepsilon$.

For each $\varepsilon$ and for any integer vector $\mathbf{i} \in \mathbb{Z}^{n}$, we shall denote by $T_{\mathbf{i}}^{\varepsilon}$ the translated image of $r(\varepsilon) T$ by the vector $\varepsilon \mathbf{i}, \mathbf{i} \in \mathbb{Z}^{n}$ :

$$
T_{\mathbf{i}}^{\varepsilon}=\varepsilon \mathbf{i}+r(\varepsilon) T
$$

Also, let us denote by $T^{\varepsilon}$ the set of all the holes contained in $\Omega$, i.e.

$$
T^{\varepsilon}=\bigcup\left\{T_{\mathbf{i}}^{\varepsilon} \mid \overline{T_{\mathbf{i}}^{\varepsilon}} \subset \Omega, \mathbf{i} \in \mathbb{Z}^{n}\right\}
$$

Set

$$
\Omega^{\varepsilon}=\Omega \backslash \overline{T^{\varepsilon}}
$$

Hence, $\Omega^{\varepsilon}$ is a periodically perforated domain with holes of the size $r(\varepsilon)$. All of them have the same shape, the distance between two adjacent holes is of order $\varepsilon$ and they do not overlap. Also, let us remark that the holes do not intersect the boundary $\partial \Omega$.

Let

$$
S^{\varepsilon}=\cup\left\{\partial T_{\mathbf{i}}^{\varepsilon} \mid \overline{T_{\mathbf{i}}^{\varepsilon}} \subset \Omega, \mathbf{i} \in \mathbb{Z}^{n}\right\}
$$

So

$$
\partial \Omega^{\varepsilon}=\partial \Omega \cup S^{\varepsilon}
$$


We shall also use the following notations:

$$
\begin{aligned}
& |\omega|=\text { the Lebesgue measure of any measurable subset } \omega \text { of } \mathbb{R}^{n}, \\
& \qquad \chi_{\omega}=\text { the characteristic function of the set } \omega, \\
& \qquad Y^{*}=Y \backslash \bar{T},
\end{aligned}
$$

and

$$
\theta=\frac{\left|Y^{*}\right|}{|Y|}
$$

Moreover, for an arbitrary function $\psi \in L^{2}\left(\Omega^{\varepsilon}\right)$, we shall denote by $\tilde{\psi}$ its extension by zero inside the holes:

$$
\tilde{\psi}=\left\{\begin{array}{lll}
\psi & \text { in } & \Omega^{\varepsilon} \\
0 & \text { in } & \Omega \backslash \overline{\Omega^{\varepsilon}}
\end{array}\right.
$$

\subsection{Setting of the problem}

As already mentioned in Introduction, we are interested in studying the behavior of solutions, in such perforated domains, of variational inequalities with highly oscillating obstacles constraints.

Define the unilateral convex set

$$
K^{\varepsilon}=\left\{v \in H^{1}\left(\Omega^{\varepsilon}\right) \mid v=0 \text { on } \partial \Omega, v \geq 0 \text { on } S^{\varepsilon}\right\} .
$$

For a given function $f \in L^{2}(\Omega)$, let us consider the following variational inequality:

$$
\left\{\begin{array}{l}
\text { Find } u^{\varepsilon} \in K^{\varepsilon} \text { such that } \\
\int_{\Omega^{\varepsilon}} D u^{\varepsilon} D\left(v^{\varepsilon}-u^{\varepsilon}\right) \mathrm{d} x \geq \int_{\Omega^{\varepsilon}} f\left(v^{\varepsilon}-u^{\varepsilon}\right) \mathrm{d} x \quad \forall v^{\varepsilon} \in K^{\varepsilon} .
\end{array}\right.
$$

Since $K^{\varepsilon}$ is a nonempty convex set, for any given $f \in L^{2}(\Omega)$, the above variational inequality has a unique solution $u^{\varepsilon} \in H^{1}\left(\Omega^{\varepsilon}\right)$ (thanks to the classical existence result of Lions and Stampacchia [22]).

We shall be interested in getting the asymptotic behavior of this solution, when $\varepsilon \rightarrow 0$. This will obviously depend on the behavior of the size $r(\varepsilon)$ of the holes. Exactly like in [9], we shall see that there exists a "critical" size of the holes that separates different possible asymptotic behaviors of our solution. This size is $r(\varepsilon) \sim \varepsilon^{n /(n-2)}$ (recall that we restrict ourselves to the case $n \geq 3$ ).

\subsection{Unilateral constraint problems in perforated domains with small holes}

In the situation of small holes, the function $r=r(\varepsilon)$ is characterized by condition (2.1). Moreover, as already mentioned in introduction, to describe the asymptotic behavior of the solution of problem (2.4), the following cases have to be distinguished:

Case 1. The size $r(\varepsilon)$ of the holes is exactly of the order of $\varepsilon^{n /(n-2)}$. That means that there exists $\delta>0$ such that

$$
\lim _{\varepsilon \rightarrow 0} r(\varepsilon) \varepsilon^{-\frac{n}{n-2}}=\delta .
$$

We shall refer to this case as being the "critical" one.

Case 2. The order of the size $r(\varepsilon)$ of the holes is smaller than the critical size, i.e. the case in which

$$
\lim _{\varepsilon \rightarrow 0} r(\varepsilon) \varepsilon^{-\frac{n}{n-2}}=0 .
$$


Case 3. The order of the size $r(\varepsilon)$ of the holes is bigger that the critical one, i.e. the case in which

$$
\lim _{\varepsilon \rightarrow 0} r(\varepsilon) \varepsilon^{-\frac{n}{n-2}}=+\infty
$$

Case 1. Let us start with the case of critical holes. Following [9], we know that for this geometry there exist a sequence of auxiliary functions $w^{\varepsilon}$ and a distribution $\mu$ such that the following hypotheses are satisfied:

(H.0) $0 \leq w^{\varepsilon} \leq 1$ a.e. in $\Omega$;

(H.1) $w^{\varepsilon} \in H^{1}(\Omega)$;

(H.2) $w^{\varepsilon}=0$ on the holes $T^{\varepsilon}$;

(H.3) $w^{\varepsilon} \rightarrow 1$ weakly in $H^{1}(\Omega)$;

(H.4) $\mu \in W^{-1, \infty}(\Omega)$;

(H.5) $\left\{\begin{array}{l}\text { For every sequence } v^{\varepsilon} \text { such that } v^{\varepsilon}=0 \text { on } T^{\varepsilon} \text { satisfying } v^{\varepsilon} \rightarrow v, \\ \text { weakly in } H^{1}(\Omega) \text {, with } v \in H^{1}(\Omega) \text {, one has } \\ \left\langle-\Delta w^{\varepsilon}, \varphi v^{\varepsilon}\right\rangle_{H^{-1}(\Omega), H_{0}^{1}(\Omega)} \rightarrow\langle\mu, \varphi v\rangle_{H^{-1}(\Omega), H_{0}^{1}(\Omega)}\end{array}\right.$ for all $\varphi \in \mathcal{D}(\Omega)$.

Under these hypotheses, let us recall that it was proved in [9] that

$$
\langle\mu, \varphi\rangle=\lim _{\varepsilon \rightarrow 0} \int_{\Omega}\left|D w^{\varepsilon}\right|^{2} \varphi \mathrm{d} x \text { for any } \varphi \in \mathcal{D}(\Omega) \text {. }
$$

So $\mu$, the limit in the distribution sense of $\left|D w^{\varepsilon}\right|^{2}$, is a positive measure.

As we know from [9], despite the fact that these hypotheses may sound a little bit strange, today they proved to be quite natural. In fact, they are verified not just for our particular geometry, but for much more general situations. In their original paper, Cioranescu and Murat made several useful remarks for a better understanding of the geometrical information contained in these hypotheses. Some of them will be very useful in what follows. For the sake of completeness, we have decided to recall them here.

Remark 2.1. If there exist $w^{\varepsilon}$ and $\mu$ satisfying hypotheses (H.0) to (H.5), they are "quasi-unique". Indeed, if we consider two pairs $\left(w^{\varepsilon}, \mu\right)$ and $\left(\overline{w^{\varepsilon}}, \bar{\mu}\right)$ satisfying hypotheses (H.0)-(H.5), one has

$$
\left\{\begin{array}{l}
\mu=\bar{\mu}, \\
w^{\varepsilon}-\overline{w^{\varepsilon}} \rightarrow 0 \text { strongly in } H_{l o c}^{1}(\Omega) .
\end{array}\right.
$$

Remark 2.2. Hypothesis (H.5) could be replaced by the following one:

$(\mathrm{H} .5)^{\prime}\left\{\begin{array}{l}\text { There exist two sequences } \mu_{\varepsilon}, \nu_{\varepsilon} \in H^{-1}(\Omega) \text { such that } \mu_{\varepsilon} \rightarrow \mu \\ \text { strongly in } H^{-1}(\Omega), \nu_{\varepsilon} \rightarrow \mu \text { weakly in } H^{-1}(\Omega) \text { and }\left\langle\nu_{\varepsilon}, v_{\varepsilon}\right\rangle=0, \\ \text { for any } v^{\varepsilon} \in H_{0}^{1}(\Omega) \text { with } v^{\varepsilon}=0 \text { on the holes } T^{\varepsilon} . \\ \text { In fact, }-\Delta w^{\varepsilon}=\mu_{\varepsilon}-\nu_{\varepsilon} \text { in } \Omega .\end{array}\right.$

Or by

$(\mathrm{H} .5)^{\prime \prime}\left\{\begin{array}{l}\text { For every sequence } v^{\varepsilon} \text { such that } v^{\varepsilon}=0 \text { on } T^{\varepsilon} \text { satisfying } v^{\varepsilon} \rightarrow v, \\ \text { weakly in } H_{0}^{1}(\Omega), \text { with } v \in H_{0}^{1}(\Omega) \text {, one has } \\ \left\langle-\Delta w^{\varepsilon}, v^{\varepsilon}\right\rangle_{H^{-1}(\Omega), H_{0}^{1}(\Omega)} \rightarrow\langle\mu, v\rangle_{H^{-1}(\Omega), H_{0}^{1}(\Omega)} .\end{array}\right.$.

Clearly, (H.5) $\Rightarrow(\text { H.5 })^{\prime \prime} \Rightarrow($ H.5) and it is proved in [9] that (H.5)' is satisfied for our special geometry.

Remark 2.3. Hypothesis (H.5)", together with (H.0), enables us to prove (2.8) for test functions $\varphi \in H_{0}^{1}(\Omega) \cap L^{\infty}(\Omega)$. 
Also, we recall the following result (for a detailed proof, see again [9]):

Proposition 2.4. Assume that hypotheses (H.1) to (H.5) are satisfied. For any sequence $z^{\varepsilon}$ and $z$ such that $z^{\varepsilon}=0$ on the holes $T^{\varepsilon}$ and $z^{\varepsilon} \rightarrow z$ weakly in $H_{0}^{1}(\Omega)$, one has

$$
\liminf _{\varepsilon \rightarrow 0} \int_{\Omega}\left|D z^{\varepsilon}\right|^{2} \mathrm{~d} x \geq \int_{\Omega}|D z|^{2} \mathrm{~d} x+\left\langle\mu, z^{2}\right\rangle .
$$

Moreover, if $z^{\varepsilon}$ satisfies

$$
\int_{\Omega}\left|D z^{\varepsilon}\right|^{2} \mathrm{~d} x \rightarrow \int_{\Omega}|D z|^{2} \mathrm{~d} x+\left\langle\mu, z^{2}\right\rangle,
$$

then

$$
z^{\varepsilon}-w^{\varepsilon} z \rightarrow 0 \text { strongly in } W_{0}^{1,1}(\Omega) .
$$

Remark 2.5. This result can be improved if one assumes that the limit $z$ is more regular, say $z \in W_{0}^{1, p}(\Omega)$, with $p>2$. For example, if $z \in C_{0}^{1}(\Omega)$, the strong convergence in $(2.11)$ takes place in $H_{0}^{1}(\Omega)$.

The main convergence result of this section (given by Th. 2.6 below) involves any extension $\widehat{u^{\varepsilon}}$ of the solution $u^{\varepsilon}$ of the variational inequality (2.4) inside the holes such that it depends continuously on $u^{\varepsilon}$ and it is positive in $T^{\varepsilon}$. This extension actually doesn't play any important role in the proof of the convergence result. In fact, for example, one could just decide to use the positive part of any classical continuous extension of $u^{\varepsilon}$. For explicit constructions of such extensions, the interested reader is referred, for example, to [8, 10]. Another possibility is to extend $u^{\varepsilon}$ inside the holes in such a way that

$$
\begin{cases}\Delta \widehat{u^{\varepsilon}}=0 & \text { in } T^{\varepsilon} \\ \widehat{u^{\varepsilon}}=u^{\varepsilon} & \text { on } S^{\varepsilon}\end{cases}
$$

Then, since $u^{\varepsilon} \in K^{\varepsilon}$, by the maximum principle we have $\widehat{u^{\varepsilon}} \geq 0$ on the holes and $\widehat{u^{\varepsilon}} \in H_{0}^{1}(\Omega)$. Also, $\left(\widehat{u^{\varepsilon}}\right)^{-}=0$ on the holes and $\left(\widehat{u^{\varepsilon}}\right)^{-} \in H_{0}^{1}\left(\Omega^{\varepsilon}\right)$.

Theorem 2.6. Under hypotheses (H.0)-(H.5), one can construct an extension $\widehat{u^{\varepsilon}}$ of the solution $u^{\varepsilon}$ of the variational inequality (2.4), positive inside the holes, such that

$$
\widehat{u^{\varepsilon}} \rightarrow u \quad \text { weakly in } H_{0}^{1}(\Omega) \text {, }
$$

where $u$ is the unique solution of the so-called homogenized problem

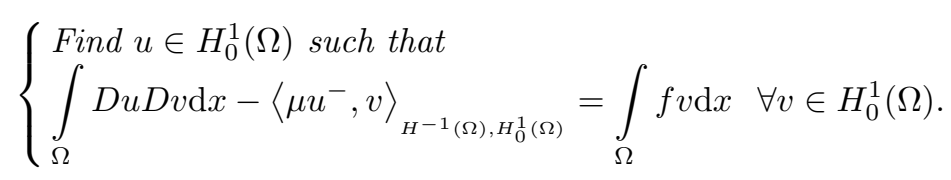

Moreover, we have the following corrector-type result

$$
\lim _{\varepsilon \rightarrow 0} \int_{\Omega^{\varepsilon}}\left|D u_{\varepsilon}^{+}-D u^{+}\right|^{2}=0
$$

and

$$
\left(\widehat{u^{\varepsilon}}\right)^{-}-w^{\varepsilon} u^{-} \rightarrow 0 \text { strongly in } W_{0}^{1,1}(\Omega)
$$


Remark 2.7. The solution of the variational inequality (2.4) is constrained to be positive on the boundary of the holes $T^{\varepsilon}$. When passing to the limit, in the regions where $u$ is nonpositive, the obstacle 0 is "seen" by $u^{\varepsilon}$ and the price to be paid is just the appearance of the penalization term $-\left\langle\mu u^{-}, v-u\right\rangle$ in the variational inequality (2.13). This fact can be better observed when looking at equations (2.14) and (2.15). Indeed, the positive part $u_{\varepsilon}^{+}$of the solution of our variational inequality does not see the oscillations of the obstacle, so we get strong convergence. Contrary, the negative part $\left(\widehat{u^{\varepsilon}}\right)^{-}$is zero inside the holes and it behaves like the solution of a variational inequality in the perforated domain $\Omega^{\varepsilon}$ with Dirichlet conditions on $\partial \Omega^{\varepsilon}$. This explains the corrector result for $\left(\widehat{u^{\varepsilon}}\right)^{-}$, which is analogous to the result obtained for the solutions of equations (see [9], Th. 3.4).

Remark 2.8. Let us note that, as in Remark 2.5, $\left(\widehat{u^{\varepsilon}}\right)^{-}-w^{\varepsilon} u^{-}$converges actually strongly to zero in a better space than $W_{0}^{1,1}(\Omega)$, namely in $W_{0}^{1, q}(\Omega), q>1$. This result can be even improved if $u^{-}$is more regular than $H_{0}^{1}(\Omega)$.

Proof of Theorem 2.6. We divide the proof into four steps.

First step. Let us introduce the following functionals defined on $H^{1}\left(\Omega^{\varepsilon}\right)$ and, respectively, $H_{0}^{1}(\Omega)$ :

$$
J^{\varepsilon}(v)=\int_{\Omega^{\varepsilon}}|D v|^{2} \mathrm{~d} x-2 \int_{\Omega^{\varepsilon}} f v \mathrm{~d} x
$$

and

$$
J^{0}(v)=\int_{\Omega}|D v|^{2} \mathrm{~d} x+\left\langle\mu,\left(v^{-}\right)^{2}\right\rangle-2 \int_{\Omega} f v \mathrm{~d} x,
$$

where the term $\left\langle\mu,\left(v^{-}\right)^{2}\right\rangle$ is the duality pairing between $\mu$, which belongs to $W^{-1, \infty}(\Omega)$ (see (H.4)) and $\left(v^{-}\right)^{2}$, which belongs to $W_{0}^{1,1}(\Omega)$, as $v^{-}$is in $H_{0}^{1}(\Omega)$.

It is well known that the solution $u^{\varepsilon}$ of the variational inequality (2.4) is also the unique solution of the minimization problem

$$
\left\{\begin{array}{l}
\text { Find } u^{\varepsilon} \in K^{\varepsilon} \text { such that } \\
J^{\varepsilon}\left(u^{\varepsilon}\right)=\inf _{v \in K^{\varepsilon}} J^{\varepsilon}(v) .
\end{array}\right.
$$

Let $v \in H_{0}^{1}(\Omega) \cap L^{\infty}(\Omega)$ be given and let us consider

$$
v^{\varepsilon}=v^{+}-w^{\varepsilon} v^{-} .
$$

Obviously, $v^{\varepsilon} \in K^{\varepsilon}$, which will allow us to take it as a test function in (2.4) and $v^{\varepsilon} \rightarrow v$ strongly in $L^{2}(\Omega)$. Now, using $v^{\varepsilon}$ in (2.4), we get:

$$
J^{\varepsilon}\left(u^{\varepsilon}\right) \leq J^{\varepsilon}\left(v^{\varepsilon}\right) .
$$

But

$$
\begin{aligned}
J^{\varepsilon}\left(v^{\varepsilon}\right)= & \int_{\Omega^{\varepsilon}}\left|D v^{+}-w^{\varepsilon} D v^{-}-v^{-} D w^{\varepsilon}\right|^{2} \mathrm{~d} x-2 \int_{\Omega^{\varepsilon}} f\left(v^{+}-w^{\varepsilon} v^{-}\right) \mathrm{d} x \\
= & \int_{\Omega^{\varepsilon}}\left|D v^{+}\right|^{2} \mathrm{~d} x+\int_{\Omega^{\varepsilon}}\left|w^{\varepsilon}\right|^{2}\left|D v^{-}\right|^{2} \mathrm{~d} x+\int_{\Omega^{\varepsilon}}\left|v^{-}\right|^{2}\left|D w^{\varepsilon}\right|^{2} \mathrm{~d} x \\
& -2 \int_{\Omega^{\varepsilon}} w^{\varepsilon} D v^{+} D v^{-} \mathrm{d} x-2 \int_{\Omega^{\varepsilon}} v^{-} D v^{+} D w^{\varepsilon} \mathrm{d} x \\
& +2 \int_{\Omega^{\varepsilon}} w^{\varepsilon} v^{-} D v^{-} D w^{\varepsilon} \mathrm{d} x-2 \int_{\Omega^{\varepsilon}} f\left(v^{+}-w^{\varepsilon} v^{-}\right) \mathrm{d} x .
\end{aligned}
$$


Due to (H.3), it is easy to pass to the limit in almost all the terms of the above identity. For the term $\int_{\Omega^{\varepsilon}}\left|v^{-}\right|^{2}\left|D w^{\varepsilon}\right|^{2} \mathrm{~d} x$, we simply use Remark 2.3. Hence, we obtain

$$
\begin{aligned}
\lim _{\varepsilon \rightarrow 0} J^{\varepsilon}\left(v^{\varepsilon}\right) & =\int_{\Omega}\left|D v^{+}\right|^{2} \mathrm{~d} x+\int_{\Omega}\left|D v^{-}\right|^{2} \mathrm{~d} x+\left\langle\mu,\left(v^{-}\right)^{2}\right\rangle-2 \int_{\Omega} f\left(v^{+}-v^{-}\right) \mathrm{d} x \\
& =\int_{\Omega}|D v|^{2} \mathrm{~d} x+\left\langle\mu,\left(v^{-}\right)^{2}\right\rangle-2 \int_{\Omega} f v \mathrm{~d} x=J^{0}(v) .
\end{aligned}
$$

So, from (2.17) we get

$$
\limsup _{\varepsilon \rightarrow 0} J^{\varepsilon}\left(u^{\varepsilon}\right) \leq J^{0}(v)
$$

for any $v \in H_{0}^{1}(\Omega) \cap L^{\infty}(\Omega)$.

Second step. Let $u^{\varepsilon} \in K^{\varepsilon}$ be the solution of the variational inequality (2.4) and let $\widehat{u^{\varepsilon}}$ be the extension of $u^{\varepsilon}$ inside the holes given by (2.12).

If we take $v^{\varepsilon}=0$, which is obviously admissible, as a test function in (2.4), we get

$$
\int_{\Omega^{\varepsilon}}\left|D u^{\varepsilon}\right|^{2} \mathrm{~d} x \leq 2 \int_{\Omega^{\varepsilon}} f u^{\varepsilon} \mathrm{d} x .
$$

Clearly,

$$
\left\|\widehat{u^{\varepsilon}}\right\|_{H_{0}^{1}(\Omega)} \leq C .
$$

Consequently, by passing to a subsequence, still denoted by $\widehat{u^{\varepsilon}}$, we can assume that there exists $u \in H_{0}^{1}(\Omega)$ such that

$$
\widehat{u^{\varepsilon}} \rightarrow u \text { weakly in } H_{0}^{1}(\Omega) \text {. }
$$

It remains to identify the limit variational inequality satisfied by $u$.

Third step. Let us decompose $\widehat{u^{\varepsilon}}$ as $\left(\widehat{u^{\varepsilon}}\right)^{+}-\left(\widehat{u^{\varepsilon}}\right)^{-}$. Obviously, since $\left(\widehat{u^{\varepsilon}}\right)^{+}$is bounded in $H_{0}^{1}(\Omega)$, it converges weakly in $H_{0}^{1}(\Omega)$ to $u^{+}$. So using the classical lower semicontinuity of the energy $\int_{\Omega}|D v|^{2} \mathrm{~d} x$, we have

$$
\liminf _{\varepsilon \rightarrow 0} \int_{\Omega^{\varepsilon}}\left|D\left(\widehat{u^{\varepsilon}}\right)^{+}\right|^{2} \mathrm{~d} x \geq \int_{\Omega}\left|D u^{+}\right|^{2} \mathrm{~d} x
$$

On the other hand, $\left(\widehat{u^{\varepsilon}}\right)^{-}$is also bounded in $H_{0}^{1}(\Omega)$ and it converges weakly in $H_{0}^{1}(\Omega)$ to $u^{-}$.

Let $\varphi \in \mathcal{D}(\Omega)$. Consider the integral

$$
X^{\varepsilon}=\int_{\Omega^{\varepsilon}}\left|D\left(\widehat{u^{\varepsilon}}\right)^{-}-\varphi D w^{\varepsilon}-w^{\varepsilon} D \varphi\right|^{2} \mathrm{~d} x
$$

By construction $D w^{\varepsilon}=w^{\varepsilon}=0$ on the holes and also, due to the fact that $\widehat{u^{\varepsilon}} \geq 0$ on the holes, $D\left(\widehat{u^{\varepsilon}}\right)^{-}=$ $\left(\widehat{u^{\varepsilon}}\right)^{-}=0$ on holes. So, we get $\left(\widehat{u^{\varepsilon}}\right)^{-} \in H_{0}^{1}\left(\Omega^{\varepsilon}\right)$ and

$$
X^{\varepsilon}=\int_{\Omega}\left|D\left(\widehat{u^{\varepsilon}}\right)^{-}-\varphi D w^{\varepsilon}-w^{\varepsilon} D \varphi\right|^{2} \mathrm{~d} x .
$$


Expanding $X^{\varepsilon}$, which is obviously nonnegative, we get

$$
\begin{aligned}
& \int_{\Omega}\left|D\left(\widehat{u^{\varepsilon}}\right)^{-}-\varphi D w^{\varepsilon}-w^{\varepsilon} D \varphi\right|^{2} \mathrm{~d} x=\int_{\Omega}\left|D\left(\widehat{u^{\varepsilon}}\right)^{-}\right|^{2} \mathrm{~d} x+\int_{\Omega}|\varphi|^{2}\left|D w^{\varepsilon}\right|^{2} \mathrm{~d} x+\int_{\Omega}\left|w^{\varepsilon}\right|^{2}|D \varphi|^{2} \mathrm{~d} x \\
& -2 \int_{\Omega} w^{\varepsilon} D\left(\widehat{u^{\varepsilon}}\right)^{-} D \varphi \mathrm{d} x-2 \int_{\Omega} D w^{\varepsilon} D\left(\widehat{u^{\varepsilon}}\right)^{-} \varphi \mathrm{d} x+2 \int_{\Omega} w^{\varepsilon} D w^{\varepsilon} \varphi D \varphi \mathrm{d} x \geq 0 .
\end{aligned}
$$

Let us extract a subsequence, still denoted by $\varepsilon$, such that

$$
\lim _{\varepsilon \rightarrow 0} \int_{\Omega}\left|D\left(\widehat{u^{\varepsilon}}\right)^{-}\right|^{2} \mathrm{~d} x=\liminf _{\varepsilon \rightarrow 0} \int_{\Omega^{\varepsilon}}\left|D\left(\widehat{u^{\varepsilon}}\right)^{-}\right|^{2} \mathrm{~d} x .
$$

Now, we can easily pass to the limit in each term of the right-hand side of (2.21), except in the fifth one. For this term, taking $\varphi\left(\widehat{u^{\varepsilon}}\right)^{-}$as a test function in the decomposition given by Remark 2.2, we have

$$
\int_{\Omega} D w^{\varepsilon} D\left(\widehat{u^{\varepsilon}}\right)^{-} \varphi \mathrm{d} x+\int_{\Omega} D w^{\varepsilon}\left(\widehat{u^{\varepsilon}}\right)^{-} D \varphi \mathrm{d} x=\left\langle\mu^{\varepsilon}, \varphi\left(\widehat{u^{\varepsilon}}\right)^{-}\right\rangle-\left\langle\nu^{\varepsilon}, \varphi\left(\widehat{u^{\varepsilon}}\right)^{-}\right\rangle .
$$

Since $\left(\widehat{u^{\varepsilon}}\right)^{-}=0$ on the holes, passing to the limit in $(2.22)$, we get

$$
\lim _{\varepsilon \rightarrow 0} \int_{\Omega} D w^{\varepsilon} D\left(\widehat{u^{\varepsilon}}\right)^{-} \varphi \mathrm{d} x=\left\langle\mu, \varphi u^{-}\right\rangle
$$

Hence, from (2.21), we obtain

$$
\liminf _{\varepsilon \rightarrow 0} \int_{\Omega^{\varepsilon}}\left|D\left(\widehat{u^{\varepsilon}}\right)^{-}\right|^{2} \mathrm{~d} x \geq 2 \int_{\Omega} D u^{-} D \varphi \mathrm{d} x-\int_{\Omega}|D \varphi|^{2} \mathrm{~d} x-\left\langle\mu, \varphi^{2}\right\rangle+2\left\langle\mu, \varphi u^{-}\right\rangle .
$$

The above inequality holds true for all $\varphi \in \mathcal{D}(\Omega)$. Let us choose $\varphi$ converging strongly to $u^{-}$in $H_{0}^{1}(\Omega)$. We have

$$
\liminf _{\varepsilon \rightarrow 0} \int_{\Omega^{\varepsilon}}\left|D\left(\widehat{u^{\varepsilon}}\right)^{-}\right|^{2} \mathrm{~d} x \geq \int_{\Omega}\left|D u^{-}\right|^{2} \mathrm{~d} x+\left\langle\mu,\left(u^{-}\right)^{2}\right\rangle
$$

Finally, from (2.20) and (2.23) we get

$$
\liminf _{\varepsilon \rightarrow 0} \int_{\Omega^{\varepsilon}}\left|D \widehat{u^{\varepsilon}}\right|^{2} \mathrm{~d} x \geq \int_{\Omega}|D u|^{2} \mathrm{~d} x+\left\langle\mu,\left(u^{-}\right)^{2}\right\rangle .
$$

On the other hand, since $\widehat{u^{\varepsilon}} \rightarrow u$ weakly in $H_{0}^{1}(\Omega)$ and strongly in $L^{2}(\Omega)$, we have

$$
\lim _{\varepsilon \rightarrow 0} \int_{\Omega^{\varepsilon}} f u^{\varepsilon} \mathrm{d} x=\lim _{\varepsilon \rightarrow 0} \int_{\Omega} f \widehat{u^{\varepsilon}} \chi_{\Omega^{\varepsilon}} \mathrm{d} x=\int_{\Omega} f u \mathrm{~d} x .
$$

Hence

$$
\liminf _{\varepsilon \rightarrow 0} J^{\varepsilon}\left(u^{\varepsilon}\right) \geq J^{0}(u) .
$$


Recalling (2.19) and (2.25), we have just proved that $u \in H_{0}^{1}(\Omega)$ satisfies

$$
\int_{\Omega}|D u|^{2} \mathrm{~d} x+\left\langle\mu,\left(u^{-}\right)^{2}\right\rangle-2 \int_{\Omega} f u \mathrm{~d} x \leq \int_{\Omega}|D v|^{2} \mathrm{~d} x+\left\langle\mu,\left(v^{-}\right)^{2}\right\rangle-2 \int_{\Omega} f v \mathrm{~d} x,
$$

for any $v \in H_{0}^{1}(\Omega) \cap L^{\infty}(\Omega)$ and hence, for any $v \in H_{0}^{1}(\Omega)$.

So the function $u$ is the unique solution of the minimization problem

$$
\left\{\begin{array}{l}
\text { Find } u \in H_{0}^{1}(\Omega) \text { such that } \\
J^{0}(u)=\inf _{v \in H_{0}^{1}(\Omega)} J^{0}(v) .
\end{array}\right.
$$

As $u$ is uniquely determined, the whole sequence $\widehat{u^{\varepsilon}}$ converges to $u$ and the first part of Theorem 2.6 is proved.

Fourth step. In order to prove the corrector result given by (2.14) and (2.15), let us notice that (2.19) implies that

$$
\limsup _{\varepsilon \rightarrow 0} J^{\varepsilon}\left(u^{\varepsilon}\right) \leq \inf _{v \in H_{0}^{1}(\Omega)} J^{0}(v)=J^{0}(u) .
$$

Then, from (2.19), (2.23) and (2.25) we get immediately

$$
\int_{\Omega^{\varepsilon}}\left|D\left(\widehat{u^{\varepsilon}}\right)^{+}\right|^{2} \mathrm{~d} x \rightarrow \int_{\Omega}\left|D u^{+}\right|^{2} \mathrm{~d} x
$$

and

$$
\int_{\Omega}\left|D\left(\widehat{u^{\varepsilon}}\right)^{-}\right|^{2} \mathrm{~d} x \rightarrow \int_{\Omega}\left|D u^{-}\right|^{2} \mathrm{~d} x+\left\langle\mu,\left(u^{-}\right)^{2}\right\rangle .
$$

From these two results, both assertions (2.14) and (2.15) can be easily obtained, the first one by classical arguments and the second one by using Proposition 2.4 (recall that $\left(\widehat{u^{\varepsilon}}\right)^{-}$vanishes on the holes). Moreover, using Poincaré's inequality, we can write

$$
\int_{\Omega^{\varepsilon}}\left|\left(\widehat{u^{\varepsilon}}\right)^{+}-u^{+}\right|^{2} \mathrm{~d} x \rightarrow 0 .
$$

This completes the proof of Theorem 2.6.

Remark 2.9. Note that we could easily get the liminf inequality (2.23) by using Proposition 2.4 , for $z^{\varepsilon}=\left(\widehat{u^{\varepsilon}}\right)^{-}$. But since we didn't provided a proof of this proposition, we preferred, for the sake of completeness, to give a detailed proof of the liminf inequality contained in (2.23).

Case 2. Let us consider now the case in which the order of the size of the holes is smaller than the critical size, i.e. the case in which $r(\varepsilon)$ satisfies (2.6). There exists an extension $\widehat{u^{\varepsilon}}$ of the solution $u^{\varepsilon}$ of the variational inequality (2.4), positive inside the holes (for example, that given by (2.12)), such that

$$
\widehat{u^{\varepsilon}} \rightarrow u \quad \text { weakly in } H_{0}^{1}(\Omega)
$$

where $u$ is the unique solution of the following limit problem

$$
\left\{\begin{array}{l}
-\Delta u=f \text { in } \Omega \\
u \in H_{0}^{1}(\Omega) .
\end{array}\right.
$$

Indeed, it is not difficult to see that in this case we get $w^{\varepsilon} \rightarrow 1$ strongly in $H^{1}(\Omega)$ and $\mu=0$. So, the holes are too small to provide any contribution ("strange term") in the limit equation. 
Case 3. Let us consider now the case in which the size of the holes is bigger than the critical one, i.e. the case in which $r(\varepsilon)$ satisfies (2.7). Again, there exists an extension $\widehat{u^{\varepsilon}}$ of the solution $u^{\varepsilon}$ of the variational inequality (2.4), positive inside the holes (for example, that given by (2.12)), such that

$$
\widehat{u^{\varepsilon}} \rightarrow u \quad \text { weakly in } H_{0}^{1}(\Omega)
$$

where $u$ is the unique solution of the following obstacle problem

$$
\left\{\begin{array}{l}
u \in H_{0}^{1}(\Omega), u \geq 0 \text { in } \Omega \\
\int_{\Omega}|D u|^{2} \mathrm{~d} x-2 \int_{\Omega} f u \mathrm{~d} x \leq \int_{\Omega}|D v|^{2} \mathrm{~d} x-2 \int_{\Omega} f v \mathrm{~d} x \quad \forall v \in H_{0}^{1}(\Omega), v \geq 0 \text { in } \Omega .
\end{array}\right.
$$

Indeed, if $v \in H_{0}^{1}(\Omega), v \geq 0$ in $\Omega$, then $v \in K^{\varepsilon}$ and

$$
J^{\varepsilon}(v)=\int_{\Omega^{\varepsilon}}|D v|^{2} \mathrm{~d} x-2 \int_{\Omega^{\varepsilon}} f v \mathrm{~d} x \rightarrow \int_{\Omega}|D v|^{2} \mathrm{~d} x-2 \int_{\Omega} f v \mathrm{~d} x,
$$

since the Lebesgue measure of $T^{\varepsilon}$ converges to zero.

On the other hand, if $u^{\varepsilon}$ is the solution of our problem then, by (2.12), there exists an extension $\widehat{u^{\varepsilon}}$ on the entire $\Omega$ such that, since $B_{C_{0} \varepsilon^{n /(n-2)}} \subset B_{r(\varepsilon)},\left(\widehat{u^{\varepsilon}}\right)^{-}=0$ on $\cup_{i} B_{C_{0} \varepsilon^{n /(n-2)}}$, for any fixed constant $C_{0}$.

Reasoning as in the first case (or simply using Prop. 2.4) and using a self-contained notation, we get

$$
\int_{\Omega}|D u|^{2} \mathrm{~d} x+\left\langle\mu_{C_{0}},\left(u^{-}\right)^{2}\right\rangle \leq \liminf _{\varepsilon \rightarrow 0} \int_{\Omega}\left|D \widehat{u^{\varepsilon}}\right|^{2} \mathrm{~d} x \leq C,
$$

for any $C_{0}$. If we let $C_{0} \rightarrow+\infty$, then $\mu_{C_{0}} \rightarrow+\infty$ and, hence, $\int_{\Omega}\left(u^{-}\right)^{2} \mathrm{~d} x \leq 0$, i.e. $u \geq 0$. This completes the proof of $(2.28)$.

\subsection{Unilateral constraint problems in perforated domains with big holes}

Recall that in this situation, the function $r=r(\varepsilon)$ satisfies the condition (2.2). As already mentioned in introduction we will refer to this situation as Case 4.

The main result of this section is the following one:

Theorem 2.10. One can construct an extension $\widehat{u^{\varepsilon}}$ of the solution $u^{\varepsilon}$ of the variational inequality (2.4), positive inside the holes, such that

$$
\widehat{u^{\varepsilon}} \rightarrow u \quad \text { weakly in } H_{0}^{1}(\Omega)
$$

where $u$ is the unique solution of

$$
\left\{\begin{array}{l}
u \in H_{0}^{1}(\Omega), u \geq 0 \quad \text { in } \Omega \\
\int_{\Omega} Q D u D u \mathrm{~d} x-2 \int_{\Omega} f u \mathrm{~d} x \leq \int_{\Omega} Q D v D v \mathrm{~d} x-2 \int_{\Omega} f v \mathrm{~d} x \quad \forall v \in H_{0}^{1}(\Omega), v \geq 0 \text { in } \Omega .
\end{array}\right.
$$

Here, $Q=\left(\left(q_{i j}\right)\right)$ is the classical homogenized matrix, whose entries are defined as follows:

$$
q_{i j}=\delta_{i j}+\frac{1}{\left|Y^{*}\right|} \int_{Y^{*}} \frac{\partial \chi_{j}}{\partial y_{i}} \mathrm{~d} y
$$


in terms of the functions $\chi_{j}, i=1, \ldots, n$, solutions of the so-called cell problems

$$
\left\{\begin{array}{l}
-\Delta \chi_{j}=0 \text { in } Y^{*} \\
\frac{\partial\left(\chi_{j}+y_{j}\right)}{\partial \nu}=0 \text { on } \partial T \\
\chi_{j} Y-\text { periodic. }
\end{array}\right.
$$

The constant matrix $Q$ is symmetric and positive-definite.

Proof. We shall not go here into the details of this proof, because in Section 4, Section 4.2, we shall provide a complete proof of a more general result, namely the homogenization of a variational inequality similar to (2.4), but for a general nonisotropic material with periodic rapidly oscillating coefficients. The scalar case will be a straightforward consequence of Theorem 4.6.

Remark 2.11. Following the same techniques as in [9], it is easy to establish the analogue of Theorem 2.6 in the case of variational inequalities of type (2.4) written for operators in divergence form $-\operatorname{div}(A \operatorname{grad})$, where $A$ is a coercive matrix, not necessarily symmetric, with entries in $L^{\infty}(\Omega)$.

Remark 2.12. It is worthwhile to remark that even if the hypotheses (H.0)-(H.5) are strongly dependent on the geometry, they are general enough to be satisfied not just for our particular framework, but for several other situations. In [9], the authors discuss various situations for which these hypotheses are satisfied. Of course, as a consequence, this means that all our previous results for Signorini's problem (2.4) hold also for the above mentioned geometries.

\section{Homogenization of Signorini's PROBlem FOR REgular materials}

In this section we will consider Signorini's problem in a similar geometry as in the previous section, but in a more general framework. The domain $\Omega^{\varepsilon}$, instead of being filled in by an homogeneous material represented by the operator $-\Delta$, will be filled in by a regular material in the sense of [11].

Let $A \in L_{\#}^{\infty}(\Omega)^{n \times n}$ be a symmetric matrix whose entries are $Y$-periodic, bounded and measurable real functions. Let us suppose that $A$ satisfies the usual coerciveness assumption: there exist two positive constants $\alpha$ and $\beta$, with $0<\alpha<\beta$, such that, for any constant vector $\xi \in \mathbb{R}^{n}$ and any $y \in Y$,

$$
\alpha|\xi|^{2} \leq A \xi \cdot \xi \leq \beta|\xi|^{2}
$$

We shall denote by $A^{\varepsilon}(x)$ the value of $A(y)$ at the point $y=x / \varepsilon$, i.e. $A^{\varepsilon}(x)=A\left(\frac{x}{\varepsilon}\right)$.

Definition 3.1. We call the material represented by the matrix $A$ regular if it admits $H^{2}$-estimates uniform in $\varepsilon$, i.e., there exists a constant $C$ independent of $\varepsilon$ such that for all given $f \in L^{2}\left(\mathbb{R}^{n}\right)$ and for any sequence $v^{\varepsilon} \rightarrow v$ weakly in $H^{1}\left(\mathbb{R}^{n}\right)$ such that

$$
-\operatorname{div}\left(A^{\varepsilon} D v^{\varepsilon}\right)=f \text { in } \mathbb{R}^{n}
$$

one has

$$
\left|v^{\varepsilon}\right|_{H^{2}\left(\mathbb{R}^{n}\right)} \leq C\|f\|_{L^{2}\left(\mathbb{R}^{n}\right)} .
$$

In [11] it is proved that smooth regular materials can be characterized as follows:

Theorem 3.2. Assume that $A \in W_{\#}^{1, \infty}(Y)^{n \times n}$. Then the following statements are equivalent:

(i) $\operatorname{div} A=0$ in $\mathbb{R}^{n}$; 
(ii) there exists a constant $C>0$, independent of $\varepsilon$, but depending on $\|A\|_{W^{1, \infty}(Y)^{n \times n}}$, such that, for all $f \in L^{2}\left(\mathbb{R}^{n}\right)$, we have

$$
\left|v^{\varepsilon}\right|_{H^{2}\left(\mathbb{R}^{n}\right)} \leq C\|f\|_{L^{2}\left(\mathbb{R}^{n}\right)} .
$$

Remark 3.3. If the matrix $A$ represents a regular material and if $\Omega$ is a bounded domain in $\mathbb{R}^{n}$, by a localization procedure (see [11]), one can easily prove that for all $f \in L^{2}(\Omega)$, for any sequence $v^{\varepsilon} \rightarrow v$ weakly in $H^{1}(\Omega)$ satisfying $-\operatorname{div}\left(A^{\varepsilon} D v^{\varepsilon}\right)=f$ in $\Omega$ and for all $\omega \subset \subset \Omega$, we have

$$
\left|v^{\varepsilon}\right|_{H^{2}(\omega)} \leq C\|f\|_{L^{2}(\omega)},
$$

where $C$ is independent of $\varepsilon$.

Remark 3.4. Recall that the function $v$ involved in the definition of a regular material is known to satisfy the so-called homogenized equation, which in this particular case reduces to

$$
-\operatorname{div}(\bar{A} D v)=f \text { in } \mathbb{R}^{n} .
$$

So the homogenized matrix is nothing else but $\bar{A}$, the average of $A$ over $Y$.

For more details and nontrivial examples of such materials, see [11].

Consider the following unilateral convex set

$$
K^{\varepsilon}=\left\{v \in H^{1}\left(\Omega^{\varepsilon}\right) \mid v=0 \text { on } \partial \Omega, v \geq 0 \text { on } S^{\varepsilon}\right\},
$$

with $\Omega^{\varepsilon}$ and $S^{\varepsilon}$ as in Section 2. For a given function $f \in L^{2}(\Omega)$, let us consider the following variational inequality:

$$
\left\{\begin{array}{l}
\text { Find } u^{\varepsilon} \in K^{\varepsilon} \text { such that } \\
\int_{\Omega^{\varepsilon}} A^{\varepsilon} D u^{\varepsilon} D\left(v^{\varepsilon}-u^{\varepsilon}\right) \mathrm{d} x \geq \int_{\Omega^{\varepsilon}} f\left(v^{\varepsilon}-u^{\varepsilon}\right) \mathrm{d} x \quad \forall v^{\varepsilon} \in K^{\varepsilon} .
\end{array}\right.
$$

Since $K^{\varepsilon}$ is a nonempty convex set, for any given $f \in L^{2}(\Omega)$, the above variational inequality has a unique solution $u^{\varepsilon} \in H^{1}\left(\Omega^{\varepsilon}\right)$.

As before, we shall be interested in getting the asymptotic behavior of this solution, when $\varepsilon \rightarrow 0$. Exactly like in Section 2, there exists a "critical" size of the holes that separates different possible asymptotic behaviors of our solution. This size is again $r(\varepsilon) \sim \varepsilon^{n /(n-2)}$. In this chapter, we shall restrict ourselves to the case of critical holes (Case 1 in Sect. 2).

The main result of this section is the following one:

Theorem 3.5. One can construct an extension $\widehat{u^{\varepsilon}}$ of the solution $u^{\varepsilon}$ of the variational inequality (3.2), positive inside the holes, such that

where $u$ is the unique solution of

$$
\widehat{u^{\varepsilon}} \rightarrow u \quad \text { weakly in } H_{0}^{1}(\Omega),
$$

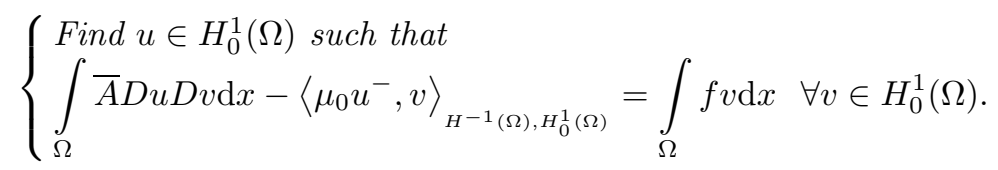

Here, $\bar{A}$ is the mean value of the matrix $A$ and it coincides, in this case, with the homogenized matrix associated to $A^{\varepsilon}$ and

$$
\mu_{0}=\inf _{w \in H^{1}\left(\mathbb{R}^{n}\right)}\left\{\int_{\mathbb{R}^{n}} A(0) D w D w \mathrm{~d} x \mid w \geq 1 \text { q.e. on } T\right\} .
$$


Moreover, we have the following corrector-type result:

$$
\lim _{\varepsilon \rightarrow 0} \int_{\Omega^{\varepsilon}}\left|D u_{\varepsilon}^{+}-D u^{+}\right|^{2}=0
$$

and

as $\varepsilon \rightarrow 0$ and $N \rightarrow \infty$.

$$
\left(\widehat{u^{\varepsilon}}\right)^{-}-w_{N}^{\varepsilon} u^{-} \rightarrow 0 \quad \text { strongly in } W_{0}^{1,1}(\Omega),
$$

In order to prove this theorem, we will need some preliminary calculations and we start by introducing some new notations. Let $Y_{\varepsilon}=\varepsilon Y$ denote the periodicity cell and let $N \in \mathbb{N}, N>1$. Let $w_{N}^{\varepsilon}$ be the unique solution of the following minimization problem on $Y_{\varepsilon}$ :

$$
\min _{w \in H^{1}\left(Y_{\varepsilon}\right)}\left\{\int_{Y_{\varepsilon}} A^{\varepsilon} D w D w \mathrm{~d} x \mid w=0 \text { in } T^{\varepsilon}, w=1 \text { on } Y_{\varepsilon} \backslash B_{N r(\varepsilon)}\right\}
$$

where $B_{N r(\varepsilon)}$ is the ball of radius $N r(\varepsilon)$ and center 0 included in $Y_{\varepsilon}$. We extend $w_{N}^{\varepsilon}$ by $\varepsilon$-periodicity to all of $\mathbb{R}^{n}$.

Let us compute $\int_{\Omega} A^{\varepsilon} D w_{N}^{\varepsilon} D w_{N}^{\varepsilon} \mathrm{d} x$. We have

$$
\int_{\Omega} A^{\varepsilon} D w_{N}^{\varepsilon} D w_{N}^{\varepsilon} \mathrm{d} x \simeq \frac{|\Omega|}{\varepsilon^{n}} \int_{Y_{\varepsilon}} A^{\varepsilon} D w_{N}^{\varepsilon} D w_{N}^{\varepsilon} \mathrm{d} x
$$

since the number of cells $Y_{\varepsilon}^{i}$ included in $\Omega$ is equivalent to $|\Omega| / \varepsilon^{n}$. Hence

$$
\int_{\Omega} A^{\varepsilon} D w_{N}^{\varepsilon} D w_{N}^{\varepsilon} \mathrm{d} x \simeq \frac{|\Omega|}{\varepsilon^{n}} \min _{w \in H^{1}\left(Y_{\varepsilon}\right)}\left\{\int_{B_{N r(\varepsilon)}} A^{\varepsilon} D w D w \mathrm{~d} x \mid w=0 \text { in } T^{\varepsilon}, w=1 \text { on } \partial B_{N r(\varepsilon)}\right\} .
$$

By changing the scale, $x=r(\varepsilon) y$, we get

$$
\int_{\Omega} A^{\varepsilon} D w_{N}^{\varepsilon} D w_{N}^{\varepsilon} \mathrm{d} x \simeq r(\varepsilon)^{n-2} \frac{|\Omega|}{\varepsilon^{n}} \min _{w \in H^{1}\left(B_{N}(0)\right)}\left\{\int_{B_{N}} A\left(\frac{r(\varepsilon)}{\varepsilon} y\right) D w D w \mathrm{~d} y \mid w=0 \text { in } T, w=1 \text { on } \partial B_{N}\right\},
$$

where we notice that the hole $r(\varepsilon) T$ has been transformed into the elementary hole $T$.

Let us introduce the sequence $\bar{\mu}_{N}^{\varepsilon}$ of positive Radon measures on $\Omega$ defined by

$$
\left\langle\bar{\mu}_{N}^{\varepsilon}, \varphi\right\rangle=\int_{\Omega} A^{\varepsilon} D w_{N}^{\varepsilon} D w_{N}^{\varepsilon} \varphi \mathrm{d} x
$$

for any $\varphi \in C_{0}^{0}(\Omega)$.

Lemma 3.6. Assume that the matrix $A$ is continuous. Then the sequence $\bar{\mu}_{N}^{\varepsilon}$ converges weakly ${ }^{*}$ in the space of Radon's measures on $\Omega$ to a limit measure $\bar{\mu}_{N}$ defined, for any $\varphi \in C_{0}^{0}(\Omega)$, by

$$
\left\langle\bar{\mu}_{N}, \varphi\right\rangle=\mu_{N} \int_{\Omega} \varphi \mathrm{d} x
$$


where

$$
\mu_{N}=\inf _{w \in H^{1}\left(B_{N}(0)\right)}\left\{\int_{B_{N}(0)} A(0) D w D w \mathrm{~d} x \mid w=0 \text { in } T, w=1 \text { on } \partial B_{N}\right\} .
$$

Proof. By the continuity of the matrix $A$, we have that

$$
\mu_{N}^{\varepsilon}=\min _{w \in H^{1}\left(B_{N}(0)\right)}\left\{\int_{B_{N}} A\left(\frac{r(\varepsilon)}{\varepsilon} y\right) D w D w \mathrm{~d} y \mid w=0 \text { on } T, w=1 \text { on } \partial B_{N}\right\}
$$

converges to $\mu_{N}$, as $\varepsilon \rightarrow 0$.

Since we are in the critical case, the coefficient in front of the definition of $\mu_{N}^{\varepsilon}$ remains bounded, as $\varepsilon \rightarrow 0$. Moreover, without loss of generality, one can choose this coefficient to be constant equal to one and we will do that in what follows inside the proof (we can choose, in fact, $\delta$ in $(2.5)$ such that $\lim _{\varepsilon \rightarrow 0} \frac{r(\varepsilon)^{n-2}}{\varepsilon^{n}}|\Omega|=1$ ). Then

$$
\left|\left\langle\bar{\mu}_{N}^{\varepsilon}, \varphi\right\rangle\right| \leq C\|\varphi\|_{C_{0}^{0}(\bar{\Omega})},
$$

for any $\varphi \in C_{0}^{0}(\Omega)$, i.e. the sequence $\bar{\mu}_{N}^{\varepsilon}$ remains bounded as $\varepsilon \rightarrow 0$ in the space of Radon's measures on $\Omega$. We can therefore extract a subsequence, still denoted by $\bar{\mu}_{N}^{\varepsilon}$, weakly * convergent to a positive measure which, thanks to the continuity of $A$, is obviously $\bar{\mu}_{N}$, defined by (3.8). Since $\bar{\mu}_{N}$ has been identified, this means that the whole sequence $\bar{\mu}_{N}^{\varepsilon}$ converges to $\bar{\mu}_{N}$. This ends the proof of Lemma 3.6.

Our next goal is to let $N \rightarrow+\infty$. Obviously, we get

$$
\lim _{N \rightarrow \infty} \bar{\mu}_{N}=\bar{\mu}_{0}
$$

where, for any $\varphi \in C_{0}^{0}(\Omega)$,

$$
\left\langle\bar{\mu}_{0}, \varphi\right\rangle=\mu_{0} \int_{\Omega} \varphi \mathrm{d} x
$$

and

$$
\mu_{0}=\inf _{w \in H^{1}\left(\mathbb{R}^{n}\right)}\left\{\int_{\mathbb{R}^{n}} A(0) D w D w \mathrm{~d} x \mid w \geq 1 \text { q.e. on } T\right\} .
$$

An immediate consequence of Lemma 3.6 is the fact that the sequence $w_{N}^{\varepsilon}$ is bounded in $H^{1}(\Omega)$, and hence relatively compact in $L^{2}(\Omega)$. Introducing $\chi_{\varepsilon}$ equal to one on $\bigcup_{i}\left(Y_{\varepsilon}^{i} \backslash B_{N r(\varepsilon)}^{i}\right)$ and zero elsewhere, we have

$$
\left(w_{N}^{\varepsilon}-1\right) \chi_{\varepsilon}=0 \text { on } \mathbb{R}^{n}
$$

and it is not difficult to see that $w_{N}^{\varepsilon} \rightarrow 1$ strongly in $L^{2}(\Omega)$.

Remark 3.7. Exactly like in Section 2, Section 2.1, using the coercivity of the matrix $A$ and arguing like in [9], Lemma 2.8, one can prove that there exist two sequences $\lambda_{N}^{\varepsilon}, \nu_{N}^{\varepsilon} \in H^{-1}(\Omega)$ such that $\lambda_{N}^{\varepsilon} \rightarrow \bar{\mu}_{N}$ strongly in $H^{-1}(\Omega), \nu_{N}^{\varepsilon} \rightarrow \bar{\mu}_{N}$ weakly in $H^{-1}(\Omega)$ and $\left\langle\nu_{N}^{\varepsilon}, v_{\varepsilon}\right\rangle=0$, for any $v^{\varepsilon} \in H_{0}^{1}(\Omega)$ with $v^{\varepsilon}=0$ on the holes $T^{\varepsilon}$. In fact,

$$
-\operatorname{div}\left(A^{\varepsilon} D w_{N}^{\varepsilon}\right)=\lambda_{N}^{\varepsilon}-\nu_{N}^{\varepsilon} \quad \text { in } \quad \Omega .
$$


Proof of Theorem 3.5. We shall divide the proof into four steps.

First step. Let us introduce the following functionals defined on $H_{0}^{1}\left(\Omega^{\varepsilon}\right)$ and, respectively, $H_{0}^{1}(\Omega)$ :

$$
J^{\varepsilon}(v)=\int_{\Omega^{\varepsilon}} A^{\varepsilon} D v D v \mathrm{~d} x-2 \int_{\Omega^{\varepsilon}} f v \mathrm{~d} x
$$

and

$$
J^{0}(v)=\int_{\Omega} \bar{A} D v D v \mathrm{~d} x+\left\langle\mu_{0},\left(v^{-}\right)^{2}\right\rangle-2 \int_{\Omega} f v \mathrm{~d} x .
$$

Let $v \in \mathcal{D}(\Omega)$ be given and, for a fixed $N$, let us consider

$$
v^{\varepsilon}=v^{+}-w_{N}^{\varepsilon} v^{-},
$$

with $w_{N}^{\varepsilon}$ defined by (3.6). Obviously, $v^{\varepsilon} \in K^{\varepsilon}$ which will allow us to take it as a test function in (3.2) and $v^{\varepsilon} \rightarrow v$ strongly in $L^{2}(\Omega)$. Using $v^{\varepsilon}$ in (3.2), we get

$$
J^{\varepsilon}\left(u^{\varepsilon}\right) \leq J^{\varepsilon}\left(v^{\varepsilon}\right) .
$$

Let us compute $J^{\varepsilon}\left(v^{\varepsilon}\right)$. We have

$$
\begin{aligned}
J^{\varepsilon}\left(v^{\varepsilon}\right)= & \int_{\Omega^{\varepsilon}} A^{\varepsilon} D\left(v^{+}-w_{N}^{\varepsilon} v^{-}\right) D\left(v^{+}-w_{N}^{\varepsilon} v^{-}\right) \mathrm{d} x-2 \int_{\Omega^{\varepsilon}} f\left(v^{+}-w_{N}^{\varepsilon} v^{-}\right) \mathrm{d} x \\
= & \int_{\Omega^{\varepsilon}} A^{\varepsilon} D v^{+} D v^{+} \mathrm{d} x+\int_{\Omega^{\varepsilon}} A^{\varepsilon}\left(w_{N}^{\varepsilon}\right)^{2} D v^{-} D v^{-} \mathrm{d} x+\int_{\Omega^{\varepsilon}} A^{\varepsilon} D w_{N}^{\varepsilon} D w_{N}^{\varepsilon}\left(v^{-}\right)^{2} \mathrm{~d} x-2 \int_{\Omega^{\varepsilon}} A^{\varepsilon} w_{N}^{\varepsilon} D v^{+} D v^{-} \mathrm{d} x \\
& -2 \int_{\Omega^{\varepsilon}} A^{\varepsilon} v^{-} D v^{+} D w_{N}^{\varepsilon} \mathrm{d} x+2 \int_{\Omega^{\varepsilon}} A^{\varepsilon} w_{N}^{\varepsilon} v^{-} D v^{-} D w_{N}^{\varepsilon} \mathrm{d} x-2 \int_{\Omega^{\varepsilon}} f\left(v^{+}-w_{N}^{\varepsilon} v^{-}\right) \mathrm{d} x
\end{aligned}
$$

Due to our hypotheses, it is easy to pass to the limit in almost all the terms of (3.16). For the term $\int_{\Omega^{\varepsilon}} A^{\varepsilon} D w_{N}^{\varepsilon} D w_{N}^{\varepsilon}\left|v^{-}\right|^{2} \mathrm{~d} x$ we use Lemma 3.5 and the fact that $\left(v^{-}\right)^{2}$ is a continuous function with compact support. For the sixth term we use the same procedure we have already used in the proof of the limsup inequality for the case of $-\Delta$, i.e. to take $w_{N}^{\varepsilon}\left(v^{-}\right)^{2}$ as a test function in the decomposition of $-\operatorname{div}\left(A^{\varepsilon} D w_{N}^{\varepsilon}\right)$ given in Remark 3.7 (or we can use the fact that $\xi$, the weak limit in $L^{2}(\Omega)$ of $A^{\varepsilon} D w_{N}^{\varepsilon}$, is zero).

Hence, taking the supremum in $N$, we have

$$
\begin{aligned}
\lim _{\varepsilon \rightarrow 0} J^{\varepsilon}\left(v^{\varepsilon}\right) & =\int_{\Omega} \bar{A} D v^{+} D v^{+} \mathrm{d} x+\int_{\Omega} \bar{A} D v^{-} D v^{-} \mathrm{d} x+\left\langle\mu_{0},\left(v^{-}\right)^{2}\right\rangle-2 \int_{\Omega} f\left(v^{+}-v^{-}\right) \mathrm{d} x \\
& =\int_{\Omega} \bar{A} D v D v \mathrm{~d} x+\left\langle\mu 0,\left(v^{-}\right)^{2}\right\rangle-2 \int_{\Omega} f v \mathrm{~d} x=J^{0}(v) .
\end{aligned}
$$

So, from (3.15) we get

$$
\limsup _{\varepsilon \rightarrow 0} J^{\varepsilon}\left(u^{\varepsilon}\right) \leq J^{0}(v)
$$

for any $v \in \mathcal{D}(\Omega)$.

Second step. Let $u^{\varepsilon} \in K^{\varepsilon}$ be the solution of the variational inequality (3.2) and, as before, let $\widehat{u^{\varepsilon}}$ be the extension of $u^{\varepsilon}$ inside the holes given by (2.12). Clearly,

$$
\left\|\widehat{u^{\varepsilon}}\right\|_{H_{0}^{1}(\Omega)} \leq C .
$$


Consequently, by passing to a subsequence, still denoted by $\widehat{u^{\varepsilon}}$, we can assume that there exists $u \in H_{0}^{1}(\Omega)$ such that

$$
\widehat{u^{\varepsilon}} \rightarrow u \text { weakly in } H_{0}^{1}(\Omega) .
$$

It remains to identify the limit variational inequality satisfied by $u$.

Third step. Let us decompose $\widehat{u^{\varepsilon}}$ as $\left(\widehat{u^{\varepsilon}}\right)^{+}-\left(\widehat{u^{\varepsilon}}\right)^{-}$. Obviously, since $\left(\widehat{u^{\varepsilon}}\right)^{+}$is bounded in $H_{0}^{1}(\Omega)$, it converges weakly in $H_{0}^{1}(\Omega)$ to $u^{+}$.

By the classical lower semicontinuity of the energy, we have

$$
\liminf _{\varepsilon \rightarrow 0} \int_{\Omega} A^{\varepsilon} D\left(\widehat{u^{\varepsilon}}\right)^{+} D\left(\widehat{u^{\varepsilon}}\right)^{+} \mathrm{d} x \geq \int_{\Omega} \bar{A} D u^{+} D u^{+} \mathrm{d} x
$$

On the other hand, $\left(\widehat{u^{\varepsilon}}\right)^{-}$is also bounded in $H_{0}^{1}(\Omega)$ and it converges weakly in $H_{0}^{1}(\Omega)$ to $u^{-}$.

Let $\varphi \in \mathcal{D}(\Omega)$. Consider, for fixed $N$, the integral

$$
X^{\varepsilon}=\int_{\Omega^{\varepsilon}} A^{\varepsilon}\left(D u_{\varepsilon}^{-}-\varphi D w_{N}^{\varepsilon}-w_{N}^{\varepsilon} D \varphi\right)\left(D u_{\varepsilon}^{-}-\varphi D w_{N}^{\varepsilon}-w_{N}^{\varepsilon} D \varphi\right) \mathrm{d} x .
$$

By construction $D w_{N}^{\varepsilon}=w_{N}^{\varepsilon}=0$ on the holes and also, due to the fact that $\widehat{u^{\varepsilon}} \geq 0$ on the holes, $D\left(\widehat{u^{\varepsilon}}\right)^{-}=$ $\left(\widehat{u^{\varepsilon}}\right)^{-}=0$ on holes. So, we get $\left(\widehat{u^{\varepsilon}}\right)^{-} \in H_{0}^{1}\left(\Omega^{\varepsilon}\right)$ and

$$
X^{\varepsilon}=\int_{\Omega} A^{\varepsilon}\left(D\left(\widehat{u^{\varepsilon}}\right)^{-}-\varphi D w_{N}^{\varepsilon}-w_{N}^{\varepsilon} D \varphi\right)\left(D\left(\widehat{u^{\varepsilon}}\right)^{-}-\varphi D w_{N}^{\varepsilon}-w_{N}^{\varepsilon} D \varphi\right) \mathrm{d} x .
$$

Expanding $X^{\varepsilon}$, which is obviously nonnegative, we get

$$
\begin{array}{r}
\int_{\Omega} A^{\varepsilon} D\left(\widehat{u^{\varepsilon}}\right)^{-} D\left(\widehat{u^{\varepsilon}}\right)^{-} \mathrm{d} x+\int_{\Omega} A^{\varepsilon} \varphi^{2} D w_{N}^{\varepsilon} D w_{N}^{\varepsilon} \mathrm{d} x+\int_{\Omega} A^{\varepsilon}\left|w_{N}^{\varepsilon}\right|^{2} D \varphi D \varphi \mathrm{d} x-2 \int_{\Omega} A^{\varepsilon} w_{N}^{\varepsilon} D\left(\widehat{u^{\varepsilon}}\right)^{-} D \varphi \mathrm{d} x \\
-2 \int_{\Omega} A^{\varepsilon} D w_{N}^{\varepsilon} D\left(\widehat{u^{\varepsilon}}\right)^{-} \varphi \mathrm{d} x+2 \int_{\Omega} A^{\varepsilon} w_{N}^{\varepsilon} D w_{N}^{\varepsilon} \varphi D \varphi \mathrm{d} x \geq 0 .
\end{array}
$$

Let us extract a subsequence, still denoted by $\varepsilon$, such that

$$
\lim _{\varepsilon \rightarrow 0} \int_{\Omega} A^{\varepsilon} D\left(\widehat{u^{\varepsilon}}\right)^{-} D\left(\widehat{u^{\varepsilon}}\right)^{-} \mathrm{d} x=\liminf _{\varepsilon \rightarrow 0} \int_{\Omega} A^{\varepsilon} D\left(\widehat{u^{\varepsilon}}\right)^{-} D\left(\widehat{u^{\varepsilon}}\right)^{-} \mathrm{d} x .
$$

We can easily pass to the limit in each term of the left-hand side of (3.19), except in the first, the fourth and the fifth ones. We intent, in fact, to evaluate the liminf of the first term. So it will be enough to evaluate the limit of the fourth and the fifth term.

For the term $-2 \int_{\Omega} A^{\varepsilon} D w_{N}^{\varepsilon} D\left(\widehat{u^{\varepsilon}}\right)^{-} \varphi \mathrm{d} x$, taking $\varphi\left(\widehat{u^{\varepsilon}}\right)^{-}$as a test function in the decomposition given in Remark 3.7, we have

$$
\int_{\Omega} A^{\varepsilon} D w_{N}^{\varepsilon} D\left(\widehat{u^{\varepsilon}}\right)^{-} \varphi \mathrm{d} x+\int_{\Omega} A^{\varepsilon} D w_{N}^{\varepsilon}\left(\widehat{u^{\varepsilon}}\right)^{-} D \varphi \mathrm{d} x=\left\langle\lambda_{N}^{\varepsilon}, \varphi\left(\widehat{u^{\varepsilon}}\right)^{-}\right\rangle-\left\langle\nu_{N}^{\varepsilon}, \varphi\left(\widehat{u^{\varepsilon}}\right)^{-}\right\rangle .
$$

Since $\left(\widehat{u^{\varepsilon}}\right)^{-}=0$ on the holes, passing to the limit in the above equality, we get

$$
\lim _{\varepsilon \rightarrow 0} \int_{\Omega} A^{\varepsilon} D w_{N}^{\varepsilon} D\left(\widehat{u^{\varepsilon}}\right)^{-} \varphi \mathrm{d} x=\left\langle\mu_{N}, \varphi u^{-}\right\rangle,
$$


since

$$
\lim _{\varepsilon \rightarrow 0} \int_{\Omega} A^{\varepsilon} D w_{N}^{\varepsilon}\left(\widehat{u^{\varepsilon}}\right)^{-} D \varphi \mathrm{d} x=0 .
$$

Indeed, if we denote the weak limit in $L^{2}(\Omega)$ of $A^{\varepsilon} D w_{N}^{\varepsilon}$ by $\xi$, then

$$
\int_{\Omega} A^{\varepsilon} D w_{N}^{\varepsilon} \varphi \mathrm{d} x \rightarrow \int_{\Omega} \xi \varphi \mathrm{d} x
$$

and also, due to the fact that we are dealing with regular materials, a simple application of the classical compensated compactness lemma shows that

$$
\int_{\Omega} A^{\varepsilon} D w_{N}^{\varepsilon} \varphi \mathrm{d} x \rightarrow 0
$$

So $\xi=0$ and consequently $\lim _{\varepsilon \rightarrow 0} \int_{\Omega} A^{\varepsilon} D w_{N}^{\varepsilon}\left(\widehat{u^{\varepsilon}}\right)^{-} D \varphi \mathrm{d} x=0$. Hence (3.20) is proved.

Let us look at the fourth term in (3.19).

$$
\begin{aligned}
\int_{\Omega} A^{\varepsilon} w_{N}^{\varepsilon} D\left(\widehat{u^{\varepsilon}}\right)^{-} D \varphi \mathrm{d} x & =-\int_{\Omega} A^{\varepsilon} D w_{N}^{\varepsilon}\left(\widehat{u^{\varepsilon}}\right)^{-} D \varphi \mathrm{d} x-\int_{\Omega} A^{\varepsilon} w_{N}^{\varepsilon}\left(\widehat{u^{\varepsilon}}\right)^{-} \Delta \varphi \mathrm{d} x \rightarrow-\int_{\Omega} \xi u^{-} D \varphi \mathrm{d} x-\int_{\Omega} \bar{A} u^{-} \Delta \varphi \mathrm{d} x \\
& =\int_{\Omega} \bar{A} D u^{-} D \varphi \mathrm{d} x .
\end{aligned}
$$

Hence, from (3.19), taking the supremum in $N$, we obtain

$$
\liminf _{\varepsilon \rightarrow 0} \int_{\Omega^{\varepsilon}} A^{\varepsilon} D\left(\widehat{u^{\varepsilon}}\right)^{-} D\left(\widehat{u^{\varepsilon}}\right)^{-} \mathrm{d} x \geq 2 \int_{\Omega} \bar{A} D u^{-} D \varphi \mathrm{d} x-\int_{\Omega} \bar{A} D \varphi D \varphi \mathrm{d} x-\left\langle\mu_{0}, \varphi^{2}\right\rangle+2\left\langle\mu_{0}, \varphi u^{-}\right\rangle .
$$

The above inequality holds true for all $\varphi \in \mathcal{D}(\Omega)$. Let us choose $\varphi$ converging strongly to $u^{-}$in $H_{0}^{1}(\Omega)$. We have

$$
\liminf _{\varepsilon \rightarrow 0} \int_{\Omega} A^{\varepsilon} D\left(\widehat{u^{\varepsilon}}\right)^{-} D\left(\widehat{u^{\varepsilon}}\right)^{-} \mathrm{d} x \geq \int_{\Omega} \bar{A} D u^{-} D u^{-} \mathrm{d} x+\left\langle\mu_{0},\left(u^{-}\right)^{2}\right\rangle .
$$

Finally, from (3.18) and (3.22) we get

$$
\liminf _{\varepsilon \rightarrow 0} \int_{\Omega} A^{\varepsilon} D \widehat{u^{\varepsilon}} \widehat{D u^{\varepsilon}} \mathrm{d} x \geq \int_{\Omega} \bar{A} D u D u \mathrm{~d} x+\left\langle\mu_{0},\left(u^{-}\right)^{2}\right\rangle .
$$

On the other hand, since $\widehat{u^{\varepsilon}} \rightarrow u$ weakly in $H_{0}^{1}(\Omega)$ and strongly in $L^{2}(\Omega)$, we have

$$
\lim _{\varepsilon \rightarrow 0} \int_{\Omega^{\varepsilon}} f u^{\varepsilon} \mathrm{d} x=\lim _{\varepsilon \rightarrow 0} \int_{\Omega} f \widehat{u^{\varepsilon}} \chi_{\Omega^{\varepsilon}} \mathrm{d} x=\int_{\Omega} f u \mathrm{~d} x .
$$

Hence

$$
\liminf _{\varepsilon \rightarrow 0} J^{\varepsilon}\left(u^{\varepsilon}\right) \geq J^{0}(u) .
$$


Recalling (3.17) and (3.25), we have just proved that $u \in H_{0}^{1}(\Omega)$ satisfies

$$
\int_{\Omega} \bar{A} D u D u \mathrm{~d} x+\left\langle\mu_{0},\left(u^{-}\right)^{2}\right\rangle-2 \int_{\Omega} f u \mathrm{~d} x \leq \int_{\Omega} \bar{A} D v D v \mathrm{~d} x+\left\langle\mu_{0},\left(v^{-}\right)^{2}\right\rangle-2 \int_{\Omega} f v \mathrm{~d} x
$$

for any $v \in \mathcal{D}(\Omega)$ and hence, by density, for any $v \in H_{0}^{1}(\Omega)$. So, the function $u$ is the unique solution of the minimization problem

$$
\left\{\begin{array}{l}
\text { Find } u \in H_{0}^{1}(\Omega) \text { such that } \\
J^{0}(u)=\inf _{v \in H_{0}^{1}(\Omega)} J^{0}(v) .
\end{array}\right.
$$

As $u$ is uniquely determined, the whole sequence $\widehat{u^{\varepsilon}}$ converges to $u$ and the third step of the proof is finished.

Fourth step. To conclude the proof of Theorem 3.5 we just need to get the corrector result given by (3.4) and (3.5). From the results of the previous step, we have immediately

$$
\int_{\Omega^{\varepsilon}} A^{\varepsilon} D\left(\widehat{u^{\varepsilon}}\right)^{+} D\left(\widehat{u^{\varepsilon}}\right)^{+} \mathrm{d} x \rightarrow \int_{\Omega} \bar{A} D u^{+} D u^{+} \mathrm{d} x
$$

and

$$
\int_{\Omega} A^{\varepsilon} D\left(\widehat{u^{\varepsilon}}\right)^{-} D\left(\widehat{u^{\varepsilon}}\right)^{-} \mathrm{d} x \rightarrow \int_{\Omega} \bar{A} D u^{-} D u^{-} \mathrm{d} x+\left\langle\mu_{0},\left(u^{-}\right)^{2}\right\rangle \cdot
$$

From these two convergences, both assertions (3.4) and (3.5) can be easily obtained, the first one by classical arguments and the second one by stating the following proposition whose proof will be given later on and which generalize Proposition 2.4 to the case of regular materials.

Proposition 3.8. Let $z^{\varepsilon}$ be any given sequence in $H_{0}^{1}(\Omega)$ such that $z^{\varepsilon}=0$ on the holes $T^{\varepsilon}$ and $z^{\varepsilon}$ converges weakly in $H_{0}^{1}(\Omega)$ to a given element $z$. If $z^{\varepsilon}$ satisfies

$$
\int_{\Omega} A^{\varepsilon} D z^{\varepsilon} D z^{\varepsilon} \mathrm{d} x \rightarrow \int_{\Omega} \bar{A} D z D z \mathrm{~d} x+\left\langle\mu_{0}, z^{2}\right\rangle
$$

then

$$
z^{\varepsilon}-w_{N}^{\varepsilon} z \rightarrow 0 \quad \text { strongly in } W_{0}^{1,1}(\Omega)
$$

for $\varepsilon \rightarrow 0$ and $N \rightarrow \infty$.

From (3.27) we see that the hypotheses of Proposition 3.8 are fulfilled by choosing $z^{\varepsilon}=\left(\widehat{u^{\varepsilon}}\right)^{-}$. Then the corrector result $(3.5)$ for $\left(\widehat{u^{\varepsilon}}\right)^{-}$is nothing else but the conclusion of the above mentioned proposition. We conclude by proving Proposition 3.8 .

Proof of of Proposition 3.8. The proof follows step by step the proof of Proposition 2.4 (see Cioranescu and Murat [9]). Let $\varphi \in \mathcal{D}(\Omega)$. We begin by developing the following expression

$$
X^{\varepsilon}=\int_{\Omega} A^{\varepsilon}\left(D\left(\widehat{u^{\varepsilon}}\right)^{-}-\varphi D w_{N}^{\varepsilon}-w_{N}^{\varepsilon} D \varphi\right)\left(D\left(\widehat{u^{\varepsilon}}\right)^{-}-\varphi D w_{N}^{\varepsilon}-w_{N}^{\varepsilon} D \varphi\right) \mathrm{d} x .
$$


We get

$$
\begin{aligned}
X^{\varepsilon}=\int_{\Omega} A^{\varepsilon} D\left(\widehat{u^{\varepsilon}}\right)^{-} D\left(\widehat{u^{\varepsilon}}\right)^{-} \mathrm{d} x & +\int_{\Omega} A^{\varepsilon} \varphi^{2} D w_{N}^{\varepsilon} D w_{N}^{\varepsilon} \mathrm{d} x+\int_{\Omega} A^{\varepsilon}\left|w_{N}^{\varepsilon}\right|^{2} D \varphi D \varphi \mathrm{d} x \\
& -2 \int_{\Omega} A^{\varepsilon} w_{N}^{\varepsilon} D\left(\widehat{u^{\varepsilon}}\right)^{-} D \varphi \mathrm{d} x-2 \int_{\Omega} A^{\varepsilon} D w_{N}^{\varepsilon} D\left(\widehat{u^{\varepsilon}}\right)^{-} \varphi \mathrm{d} x+2 \int_{\Omega} A^{\varepsilon} w_{N}^{\varepsilon} D w_{N}^{\varepsilon} \varphi D \varphi \mathrm{d} x .
\end{aligned}
$$

One can pass to the limit in all these six terms following the same procedure as in Step 3 . By doing this, one reach immediately the conclusion that $X^{\varepsilon} \rightarrow 0$, as $\varepsilon \rightarrow 0$ and $N \rightarrow \infty$, which ends the proof of Proposition 3.8.

\section{Homogenization of Signorini's problem for Periodic heterogeneous media}

In this section we consider the case of a general medium represented by a coercive periodic matrix with rapidly oscillating entries. The geometrical framework is the same as in the previous chapters and again we shall deal just with the two interesting geometrical cases: the critical case (Case $\mathbf{1}$ in Sect. 2) and the case of big holes, i.e., holes of the same size as the period (Case 4 in Sect. 2).

Let $A \in L_{\#}^{\infty}(\Omega)^{n \times n}$ be a symmetric matrix whose entries are $Y$-periodic, bounded and measurable real functions. Let us suppose that $A$ satisfies the usual coerciveness assumption: there exist two positive constants $\alpha$ and $\beta$, with $0<\alpha<\beta$, such that, for any constant vector $\xi \in \mathbb{R}^{n}$ and any $y \in Y$,

$$
\alpha|\xi|^{2} \leq A \xi \cdot \xi \leq \beta|\xi|^{2}
$$

We shall denote by $A^{\varepsilon}(x)$ the value of $A(y)$ at the point $y=x / \varepsilon$, i.e. $A^{\varepsilon}(x)=A\left(\frac{x}{\varepsilon}\right)$. We further assume that $A$ is continuous with respect to $y$.

For a given function $f \in L^{2}(\Omega)$, let us consider again the following variational inequality:

$$
\left\{\begin{array}{l}
\text { Find } u^{\varepsilon} \in K^{\varepsilon} \text { such that } \\
\int_{\Omega^{\varepsilon}} A^{\varepsilon} D u^{\varepsilon} D\left(v^{\varepsilon}-u^{\varepsilon}\right) \mathrm{d} x \geq \int_{\Omega^{\varepsilon}} f\left(v^{\varepsilon}-u^{\varepsilon}\right) \mathrm{d} x \quad \forall v^{\varepsilon} \in K^{\varepsilon}
\end{array}\right.
$$

with $K^{\varepsilon}$ defined in the previous chapters. As before, the above variational inequality has a unique solution $u^{\varepsilon} \in H^{1}\left(\Omega^{\varepsilon}\right)$.

\subsection{Small holes (Critical case)}

In this case, the limit of $u^{\varepsilon}$ will be the solution of a Dirichlet problem in $\Omega$ associated with a new operator which is the sum of the standard homogenized one and an extra term ("strange term") that comes in from the geometry. The limit problem takes into account all the ingredients involved in (4.1). It captures the oscillations coming both from the periodic heterogeneous structure of the medium and those one due to the critical size of the holes. The other ingredient contained in problem (4.2) below is the spreading effect of the unilateral condition $u^{\varepsilon} \geq 0$ on $S^{\varepsilon}$ which can be seen by the fact that the strange term only charges the negative part of $u$.

Theorem 4.1. There exists an extension $\widehat{u^{\varepsilon}}$ of the solution $u^{\varepsilon}$ of the variational inequality (4.1), positive inside the holes, such that

$$
\widehat{u^{\varepsilon}} \rightarrow u \quad \text { weakly in } H_{0}^{1}(\Omega) \text {, }
$$


where $u$ is the unique solution of

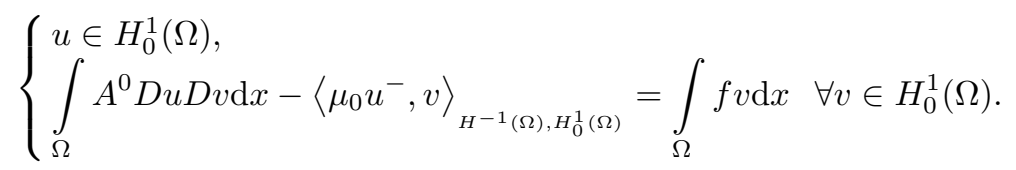

Here, $A^{0}$ is the classical homogenized matrix, whose entries are defined as follows:

$$
a_{i j}^{0}=\frac{1}{|Y|} \int_{Y}\left(a_{i j}(y)+a_{i k}(y) \frac{\partial \chi_{j}}{\partial y_{k}}\right) \mathrm{d} y
$$

in terms of the functions $\chi_{j}, j=1, \ldots, n$, solutions of the so-called cell problems

$$
\left\{\begin{array}{l}
-\operatorname{div}_{y}\left(A(y) D_{y}\left(y_{j}+\chi_{j}\right)\right)=0 \text { in } \mathbb{R}^{n} \\
\chi_{j}-Y \text { periodic }
\end{array}\right.
$$

and $\mu_{0}$ is given by

$$
\mu_{0}=\inf _{w \in H^{1}\left(\mathbb{R}^{n}\right)}\left\{\int_{\mathbb{R}^{n}} A(0) D w D w \mathrm{~d} x \mid w \geq 1 \text { q.e. on } T\right\} .
$$

The constant matrix $A^{0}$ is symmetric and positive-definite.

Remark 4.2. The results of Section 3 concerning the convergence of $\widehat{u^{\varepsilon}}$ to $u$ and the identification of $u$ can be recovered immediately from Theorem 4.1, but the proof we give here is more general.

Remark 4.3. Without loss of generality, we can consider that the holes are spheres $B_{r(\varepsilon)}(\varepsilon \mathbf{i})$ centered at the lattice points $\varepsilon \mathbf{i}$ and having the radius equal to $r(\varepsilon)$. All the results of this section can be easily extended to the case of nonspherical holes. In fact, instead of working on $B_{r(\varepsilon)}(\varepsilon \mathbf{i})$, we can replace it by $\varepsilon \mathbf{i}+r(\varepsilon) T$ and the same conclusion follows upon replacing $B_{1}(\mathbf{0})$ by $T$ in (4.15).

In order to prove Theorem 4.1, we shall use a technical result, based on the method introduced by De Giorgi [18] to match boundary conditions for minimizing sequences (see also [1] for the application of De Giorgi's method). This will allow us to modify sequences of functions near the holes $B_{r(\varepsilon)}(\varepsilon \mathbf{i})$ and to separate the contribution of the gradient of our solution close and far from the holes. By doing this, we shall be able to capture, simultaneously, the oscillations in the functional coming from the periodic oscillations of the medium and those arising from the oscillations of the boundaries of the holes.

In order to formulate this result in its full generality, let us introduce $f_{\varepsilon}: \mathbb{R}^{n} \times \mathbb{R}^{n} \rightarrow[0, \infty)$ to be a Borel function such that $f_{\varepsilon}(x, \cdot)$ is positively homogeneous of degree 2 for all $x \in \mathbb{R}^{n}$ and satisfies a growth condition of order 2 , uniformly in $\varepsilon$, i.e. there exist two strictly positive constants $C_{1}$ and $C_{2}$ such that, for any $x$ and $z$ :

$$
C_{1}|z|^{2} \leq f_{\varepsilon}(x, z) \leq C_{2}|z|^{2}
$$

We recall the above mentioned technical result (for a detailed proof, see [1]):

Lemma 4.4. Let $v^{\varepsilon}$ be a sequence weakly convergent to some $v$ in $H_{0}^{1}(\Omega)$ and let $k \in \mathbb{N}$ be fixed. Let

$$
\mathbb{Z}^{\varepsilon}=\left\{\mathbf{i} \in \mathbb{Z}^{n} \mid \operatorname{dist}\left(\varepsilon \mathbf{i}, \mathbb{R}^{n} \backslash \Omega\right)>\varepsilon\right\}
$$

and let $\delta_{\varepsilon}$ be a sequence of positive numbers with $\delta_{\varepsilon}<\varepsilon / 2$. 
For all $\mathbf{i} \in \mathbb{Z}^{\varepsilon}$, there exist $k_{\mathbf{i}} \in\{0, \ldots, k-1\}$ such that, setting

$$
\begin{gathered}
C_{\mathbf{i}}^{\varepsilon}=\left\{x \in \Omega\left|\frac{\delta_{\varepsilon}}{2^{k_{\mathbf{i}}+1}}<\right| x-\varepsilon \mathbf{i} \mid<\frac{\delta_{\varepsilon}}{2^{k_{i}}}\right\}, \\
v_{\mathbf{i}}^{\varepsilon}=\frac{1}{\left|C_{\mathbf{i}}^{\varepsilon}\right|} \int_{C_{\mathbf{i}}^{\varepsilon}} v^{\varepsilon} \mathrm{d} x \text { (the mean value of } v^{\varepsilon} \text { on } C_{\mathbf{i}}^{\varepsilon} \text { ) }
\end{gathered}
$$

and

$$
\left.\delta_{\varepsilon}^{\mathbf{i}}=\frac{3}{4} \frac{1}{2^{k_{i}}} \delta_{\varepsilon} \text { (the middle radius of } C_{\mathbf{i}}^{\varepsilon}\right),
$$

there exists a sequence $w^{\varepsilon}$, with $w^{\varepsilon} \rightarrow v$ weakly in $H_{0}^{1}(\Omega)$, such that

$$
\begin{gathered}
w^{\varepsilon}=v^{\varepsilon} \text { on } \quad \Omega \backslash \bigcup_{\mathbf{i} \in \mathbb{Z}^{\varepsilon}} C_{\mathbf{i}}^{\varepsilon}, \\
w^{\varepsilon}(x)=v_{\mathbf{i}}^{\varepsilon} \quad \text { if } \quad|x-\varepsilon \mathbf{i}|=\delta_{\varepsilon}^{\mathbf{i}}
\end{gathered}
$$

and

$$
\left|\int_{\Omega}\left(f_{\varepsilon}\left(x, D w^{\varepsilon}\right)-f_{\varepsilon}\left(x, D v^{\varepsilon}\right)\right) \mathrm{d} x\right| \leq \frac{C}{k} .
$$

Moreover, if $\delta_{\varepsilon}=o(\varepsilon)$ and the sequence $\left(\left|D v^{\varepsilon}\right|^{2}\right)$ is equi-integrable, then we can choose $k_{\mathbf{i}}=0$ for all $\mathbf{i} \in \mathbb{Z}^{\varepsilon}$.

Proof of Theorem 4.1. We divide the proof into three steps.

First step. Let $u^{\varepsilon} \in K^{\varepsilon}$ be the solution of the variational inequality (4.1) and let $\widehat{u^{\varepsilon}}$ the extension of $u^{\varepsilon}$ inside the holes given by (2.12). Clearly,

$$
\left\|\widehat{u^{\varepsilon}}\right\|_{H_{0}^{1}(\Omega)} \leq C .
$$

Consequently, by passing to a subsequence, still denoted by $\widehat{u^{\varepsilon}}$, we can assume that there exists $u \in H_{0}^{1}(\Omega)$ such that

$$
\widehat{u^{\varepsilon}} \rightarrow u \quad \text { weakly in } H_{0}^{1}(\Omega) \text {. }
$$

It remains to identify the limit variational inequality satisfied by $u$.

Second step. Let us decompose $\widehat{u^{\varepsilon}}$ as $\left(\widehat{u^{\varepsilon}}\right)^{+}-\left(\widehat{u^{\varepsilon}}\right)^{-}$. Obviously, since $\left(\widehat{u^{\varepsilon}}\right)^{+}$is bounded in $H_{0}^{1}(\Omega)$, it converges weakly in $H_{0}^{1}(\Omega)$ to $u^{+}$. We have

$$
\liminf _{\varepsilon \rightarrow 0} \int_{\Omega} A^{\varepsilon} D\left(\widehat{u^{\varepsilon}}\right)^{+} D\left(\widehat{u^{\varepsilon}}\right)^{+} \mathrm{d} x \geq \int_{\Omega} A^{0} D u^{+} D u^{+} \mathrm{d} x .
$$

It is worthwhile to notice that the contribution of $D\left(\widehat{u^{\varepsilon}}\right)^{+}$inside the holes doesn't play any role in passing to the limit because we are dealing with an equi-integrable sequence of functions and the Lebesgue measure of the holes goes to zero, as $\varepsilon \rightarrow 0$.

On the other hand, $\left(\widehat{u^{\varepsilon}}\right)^{-}$is also bounded in $H_{0}^{1}(\Omega)$ and it converges weakly in $H_{0}^{1}(\Omega)$ to $u^{-}$. Since we intent to prove that

$$
\liminf _{\varepsilon \rightarrow 0} \int_{\Omega^{\varepsilon}} A^{\varepsilon} D \widehat{u^{\varepsilon}} \overrightarrow{u^{\varepsilon}} \mathrm{d} x \geq \int_{\Omega} A^{0} D u D u \mathrm{~d} x+\left\langle\mu_{0},\left(u^{-}\right)^{2}\right\rangle
$$

it suffices to get

$$
\liminf _{\varepsilon \rightarrow 0} \int_{\Omega^{\varepsilon}} A^{\varepsilon} D\left(\widehat{u^{\varepsilon}}\right)^{-} D\left(\widehat{u^{\varepsilon}}\right)^{-} \mathrm{d} x \geq \int_{\Omega} A^{0} D u^{-} D u^{-} \mathrm{d} x+\left\langle\mu_{0},\left(u^{-}\right)^{2}\right\rangle .
$$


To this end we shall use Lemma 4.4, which obviously applies for our framework and for $f_{\varepsilon}=A^{\varepsilon} D\left(\widehat{u^{\varepsilon}}\right)^{-} D\left(\widehat{u^{\varepsilon}}\right)^{-}$. Let us fix $k$ and $N \in \mathbb{N}$, with $N>1$ and let us choose

$$
\delta_{\varepsilon}=\operatorname{Nr}(\varepsilon)
$$

Obviously, $\delta_{\varepsilon}=o(\varepsilon)$. Observe that since $\left|D\left(\widehat{u^{\varepsilon}}\right)^{-}\right|^{2}$ is equi-integrable, we can choose $k_{\mathbf{i}}=0$ for all $\mathbf{i} \in \mathbb{Z}^{\varepsilon}$. Let us set

$$
\begin{gathered}
C_{\mathbf{i}}^{\varepsilon}=\left\{x \in \Omega\left|\frac{1}{2} \operatorname{Nr}(\varepsilon)<\right| x-\varepsilon \mathbf{i} \mid<N r(\varepsilon)\right\}, \\
\overline{u_{\varepsilon \mathbf{i}}^{-}}=\frac{1}{\left|C_{\mathbf{i}}^{\varepsilon}\right|} \int_{C_{\mathbf{i}}^{\varepsilon}} u_{\varepsilon}^{-} \mathrm{d} x\left(\text { the mean value of } u_{\varepsilon}^{-} \text {on } C_{\mathbf{i}}^{\varepsilon}\right)
\end{gathered}
$$

and

$$
\delta_{\varepsilon}^{\mathbf{i}}=\frac{3}{4} \delta_{\varepsilon}\left(\text { the middle radius of } C_{\mathbf{i}}^{\varepsilon}\right) .
$$

Then, by virtue of Lemma 4.4, there exists a sequence $v^{\varepsilon}$, with $v^{\varepsilon} \rightarrow u^{-}$weakly in $H_{0}^{1}(\Omega)$, such that

$$
\begin{gathered}
v^{\varepsilon}=\left(\widehat{u^{\varepsilon}}\right)^{-} \text {on } \quad \Omega \backslash \bigcup_{\mathbf{i} \in \mathbb{Z}^{\varepsilon}} C_{\mathbf{i}}^{\varepsilon}, \\
v^{\varepsilon}(x)=\overline{u_{\varepsilon \mathbf{i}}^{-}} \text {if } \quad|x-\varepsilon \mathbf{i}|=\delta_{\varepsilon}^{\mathbf{i}}
\end{gathered}
$$

and

$$
\left|\int_{\Omega}\left(A^{\varepsilon} D v^{\varepsilon} D v^{\varepsilon}-A^{\varepsilon} D\left(\widehat{u^{\varepsilon}}\right)^{-} D\left(\widehat{u^{\varepsilon}}\right)^{-}\right) \mathrm{d} x\right| \leq \frac{C}{k} .
$$

Note that by this choice of $\delta_{\varepsilon}$ we keep $v^{\varepsilon}=\left(\widehat{u^{\varepsilon}}\right)^{-}=0$ on the holes.

Since

$$
\int_{\Omega^{\varepsilon}} A^{\varepsilon} D\left(\widehat{u^{\varepsilon}}\right)^{-} D\left(\widehat{u^{\varepsilon}}\right)^{-} \mathrm{d} x=\int_{\Omega} A^{\varepsilon} D\left(\widehat{u^{\varepsilon}}\right)^{-} D\left(\widehat{u^{\varepsilon}}\right)^{-} \mathrm{d} x
$$

using (4.8), we get

$$
\int_{\Omega} A^{\varepsilon} D\left(\widehat{u^{\varepsilon}}\right)^{-} D\left(\widehat{u^{\varepsilon}}\right)^{-} \mathrm{d} x \geq \int_{\Omega} A^{\varepsilon} D v^{\varepsilon} D v^{\varepsilon} \mathrm{d} x-\frac{C}{k} .
$$

Splitting the first term of the right-hand side, we have

$$
\int_{\Omega} A^{\varepsilon} D\left(\widehat{u^{\varepsilon}}\right)^{-} D\left(\widehat{u^{\varepsilon}}\right)^{-} \mathrm{d} x \geq \int_{\Omega \backslash F^{\varepsilon}} A^{\varepsilon} D v^{\varepsilon} D v^{\varepsilon} \mathrm{d} x+\int_{F^{\varepsilon}} A^{\varepsilon} D v^{\varepsilon} D v^{\varepsilon} \mathrm{d} x-\frac{C}{k},
$$

where

$$
F^{\varepsilon}=\bigcup_{\mathbf{i} \in \mathbb{Z}^{\varepsilon}} B_{\frac{3}{4}} N r(\varepsilon)(\varepsilon \mathbf{i})
$$

Since $\left|F^{\varepsilon}\right| \rightarrow 0$ as $\varepsilon \rightarrow 0$, we obviously have

$$
\liminf _{\varepsilon \rightarrow 0} \int_{\Omega \backslash F^{\varepsilon}} A^{\varepsilon} D v^{\varepsilon} D v^{\varepsilon} \mathrm{d} x \geq \int_{\Omega} A^{0} D u^{-} D u^{-} \mathrm{d} x
$$


So in order to get (4.7), it suffices to evaluate the liminf of $\int_{F^{\varepsilon}} A^{\varepsilon} D v^{\varepsilon} D v^{\varepsilon} \mathrm{d} x$ and to let $k \rightarrow \infty$. To this end, we will decompose the integral over $F^{\varepsilon}$ into a sum of integrals over each ball $B_{\frac{3}{4}} N r(\varepsilon)(\varepsilon \mathbf{i})$. In each of these balls, we perform the following change of variables

$$
y=\frac{x-\varepsilon \mathbf{i}}{r(\varepsilon)}
$$

Define $\zeta: B_{\frac{3}{4} N}(0) \rightarrow \mathbb{R}$ by

$$
\zeta(y)= \begin{cases}\frac{v^{\varepsilon}(\varepsilon \dot{\mathbf{i}}+r(\varepsilon) y)}{\overline{u_{\varepsilon \mathbf{i}}^{-}}} & \text {if } \overline{u_{\varepsilon \mathbf{i}}^{-}} \neq 0, \\ 0 & \text { if } \overline{u_{\varepsilon \mathbf{i}}^{-}}=0 .\end{cases}
$$

By construction, for a fixed $\mathbf{i}, \zeta \in H^{1}\left(B_{\frac{3}{4} N}(0)\right), \zeta=1$ on $\partial B_{\frac{3}{4} N}(0)$ and $\zeta=0$ on the elementary hole $B_{1}(0)$. As in Section 3, let us define, for each $x \in B_{\frac{3}{4} N r(\varepsilon)}(\varepsilon \mathbf{i})$,

$$
\mu_{0, \varepsilon}^{N}(x)=\inf _{\zeta \in H^{1}\left(B_{\frac{3}{4} N}(0)\right)}\left\{\int_{B_{\frac{3}{4} N}(0)} A\left(\frac{x}{\varepsilon}+\frac{r(\varepsilon)}{\varepsilon} y\right) D \zeta D \zeta \mathrm{d} y \mid \zeta=0 \text { on } B_{1}(0), \zeta=1 \text { on } \partial B_{\frac{3}{4} N}(0)\right\} .
$$

Let us now calculate $\int_{B_{\frac{3}{4} N r(\varepsilon)}(\varepsilon \mathbf{i})} A^{\varepsilon}(x) D v^{\varepsilon} D v^{\varepsilon} \mathrm{d} x$, which represents each of the terms in the sum giving the second term in the right-hand side of (4.9). Performing the change of variables (4.11), we have

$$
\int_{B_{\frac{3}{4} N r(\varepsilon)}(\varepsilon \mathbf{i})} A^{\varepsilon}(x) D v^{\varepsilon} D v^{\varepsilon} \mathrm{d} x=\varepsilon^{n} \int_{B_{\frac{3}{4} N}(0)} A\left(\frac{\varepsilon \mathbf{i}}{\varepsilon}+\frac{r(\varepsilon)}{\varepsilon} y\right)\left|\overline{u_{\varepsilon \mathbf{i}}^{-}}\right|^{2} D \zeta D \zeta \mathrm{d} y .
$$

So to evaluate the liminf of $\int_{F^{\varepsilon}} A^{\varepsilon} D v^{\varepsilon} D v^{\varepsilon} \mathrm{d} x$, it suffices to evaluate

$$
\liminf _{\varepsilon \rightarrow 0} \sum_{\mathbf{i} \in \mathbb{Z}^{\varepsilon}} \varepsilon^{n}\left|\overline{u_{\varepsilon \mathbf{i}}^{-}}\right|^{2} \mu_{0, \varepsilon}^{N}(\varepsilon \mathbf{i}),
$$

and, like in [1], we will do this by rewriting the sum as an integral over $\Omega$ of step functions.

Let

$$
g^{\varepsilon}(x)=\sum_{\mathbf{i} \in \mathbb{Z}^{\varepsilon}}\left|\overline{u_{\varepsilon \mathbf{i}}^{-}}\right|^{2} \chi_{Y_{\varepsilon \mathbf{i}}}(x)
$$

and

$$
\bar{\mu}_{0, \varepsilon}^{N}=\sum_{\mathbf{i} \in \mathbb{Z}^{\varepsilon}} \mu_{0, \varepsilon}^{N}(\varepsilon \mathbf{i}) \chi_{Y_{\varepsilon \mathbf{i}}} .
$$

Hence, we have to compute

$$
\liminf _{\varepsilon \rightarrow 0} \int_{\Omega} \bar{\mu}_{0, \varepsilon}^{N} g^{\varepsilon} \mathrm{d} x
$$

Notice that since $\mu_{0, \varepsilon}^{N}$ is 1-periodic we have $\mu_{0, \varepsilon}^{N}(\varepsilon \mathbf{i})=\mu_{0, \varepsilon}^{N}(0)$.

Clearly, $\bar{\mu}_{0, \varepsilon}^{N}$ is bounded in $L^{\infty}(\Omega)$, and hence, up to a subsequence, there exists $\bar{\mu}_{0}^{N}$ in $L^{\infty}(\Omega)$ such that $\bar{\mu}_{0, \varepsilon}^{N} \stackrel{*}{\rightarrow} \bar{\mu}_{0}^{N}$ weakly * in $L^{\infty}(\Omega)$. Arguing as in Lemma 3.6, it is an easy matter to identify $\bar{\mu}_{0}^{N}$. Indeed, by the continuity of the matrix $A, \bar{\mu}_{0}^{N}$ is nothing else but the measure defined, for any $\varphi \in C_{0}^{0}(\Omega)$, by

$$
\left\langle\bar{\mu}_{0}^{N}, \varphi\right\rangle=\mu_{0}^{N} \int_{\Omega} \varphi \mathrm{d} x
$$


where

$$
\mu_{0}^{N}=\inf _{\zeta \in H^{1}\left(B_{\frac{3}{4} N}(0)\right)}\left\{\int_{B_{\frac{3}{4} N}(0)} A(0) D \zeta D \zeta \mathrm{d} x \mid \zeta=0 \text { on } B_{1}(0), \zeta=1 \text { on } \partial B_{\frac{3}{4} N}(0)\right\}
$$

On the other hand, the asymptotic behavior of $g^{\varepsilon}$ is given by the following lemma, whose proof will be given later on.

Lemma 4.5. The sequence $g^{\varepsilon}$ defined by (4.12) converges strongly in $L^{1}(\Omega)$ to $\left|u^{-}\right|^{2}$.

Putting together the convergence results for $\bar{\mu}_{0, \varepsilon}^{N}$ and $g^{\varepsilon}$, we conclude

$$
\liminf _{\varepsilon \rightarrow 0} \int_{\Omega} \bar{\mu}_{0, \varepsilon}^{N} g^{\varepsilon} \mathrm{d} x=\int_{\Omega} \mu_{0}^{N}\left|u^{-}\right|^{2} \mathrm{~d} x
$$

Letting $N \rightarrow \infty$, we have

$$
\lim _{N \rightarrow \infty} \mu_{0}^{N}=\mu_{0}
$$

with

$$
\mu_{0}=\inf _{\zeta \in H^{1}\left(\mathbb{R}^{n}\right)}\left\{\int_{\mathbb{R}^{n}} A(0) D \zeta D \zeta \mathrm{d} x \mid \zeta \geq 1 \text { q.e. on } B_{1}(0)\right\} .
$$

Hence

$$
\liminf _{\varepsilon \rightarrow 0} \int_{F^{\varepsilon}} A^{\varepsilon} D v^{\varepsilon} D v^{\varepsilon} \mathrm{d} x \geq \int_{\Omega} \mu_{0}\left(u^{-}\right)^{2} \mathrm{~d} x .
$$

Using (4.9), (4.10) and (4.16), we obtain

$$
\liminf _{\varepsilon \rightarrow 0} \int_{\Omega^{\varepsilon}} A^{\varepsilon} D\left(\widehat{u^{\varepsilon}}\right)^{-} D\left(\widehat{u^{\varepsilon}}\right)^{-} \mathrm{d} x \geq \int_{\Omega} A^{0} D u^{-} D u^{-} \mathrm{d} x+\left\langle\mu_{0},\left(u^{-}\right)^{2}\right\rangle-\frac{C}{k} .
$$

Finally, letting $k \rightarrow \infty$ we get the desired liminf inequality (4.7) for $\left(\widehat{u^{\varepsilon}}\right)^{-}$. Putting together (4.5) and (4.7), we get (4.6) and this concludes this step of the proof, since the linear term in the functional $J^{\varepsilon}$ passes immediately to the limit, as $\varepsilon \rightarrow 0$.

Third step. Let $v \in H_{0}^{1}(\Omega) \cap L^{\infty}(\Omega)$ and let $v^{\varepsilon}$ such that $v^{\varepsilon} \rightarrow v$ weakly in $H_{0}^{1}(\Omega)$ and strongly in $L^{2}(\Omega)$ and $\lim _{\varepsilon \rightarrow 0} \int_{\Omega^{\varepsilon}} A^{\varepsilon} D v^{\varepsilon} D v^{\varepsilon} \mathrm{d} x=\int_{\Omega} A^{0} D v D v \mathrm{~d} x$. Moreover, we can assume that $\left|D v^{\varepsilon}\right|^{2}$ is equi-integrable. Also, by Lemma 4.4, it is not restrictive to assume that $v^{\varepsilon}=\overline{v_{\varepsilon \mathbf{i}}}$ if $|x-\varepsilon \mathbf{i}|=\frac{3}{4} \operatorname{Nr}(\varepsilon)$. Let

$$
\mu_{0, \varepsilon}^{N}(x)=\inf _{\zeta \in H^{1}\left(B_{\frac{3}{4} N}(0)\right)}\left\{\int_{B_{\frac{3}{4} N}(0)} A\left(\frac{x}{\varepsilon}+\frac{r(\varepsilon)}{\varepsilon} y\right) D \zeta D \zeta d y \mid \zeta=0 \text { on } B_{1}(0), \zeta=1 \text { on } \partial B_{\frac{3}{4} N}(0)\right\}
$$

Then, there exists $\zeta_{\mathbf{i}}^{\varepsilon} \in H^{1}\left(B_{\frac{3}{4} N}(0)\right), \zeta_{\mathbf{i}}^{\varepsilon}=0$ on $B_{1}(0), \zeta_{\mathbf{i}}^{\varepsilon}=1$ on $\partial B_{\frac{3}{4} N}(0)$ such that

$$
\int_{B_{\frac{3}{4} N}(0)} A\left(\frac{\varepsilon \mathbf{i}}{\varepsilon}+\frac{r(\varepsilon)}{\varepsilon} y\right) D \zeta_{\mathbf{i}}^{\varepsilon} D \zeta_{\mathbf{i}}^{\varepsilon} d y \leq \mu_{0, \varepsilon}^{N}(\varepsilon \mathbf{i})+\varepsilon .
$$


Define

$$
\widetilde{v^{\varepsilon}}(x)= \begin{cases}\left(v_{\varepsilon}^{+}-v_{\varepsilon}^{-}\right)(x) & \text { on } \Omega \backslash \bigcup_{\mathbf{i}} B_{\frac{3}{4}} N r(\varepsilon)(\varepsilon \mathbf{i}), \\ \overline{v_{\varepsilon \mathbf{i}}^{+}}-\overline{v_{\varepsilon \mathbf{i}}^{-}} \zeta_{\mathbf{i}}^{\varepsilon}\left(\frac{x-\varepsilon \mathbf{i}}{r(\varepsilon)}\right) & \text { on } \bigcup_{\mathbf{i}} B_{\frac{3}{4} N r(\varepsilon)}(\varepsilon \mathbf{i}) .\end{cases}
$$

Clearly $\widetilde{v^{\varepsilon}} \in K^{\varepsilon}$ and $\widetilde{v^{\varepsilon}} \rightarrow v$ strongly in $L^{2}(\Omega)$. So

$$
J^{\varepsilon}\left(u^{\varepsilon}\right) \leq J^{\varepsilon}\left(\widetilde{v^{\varepsilon}}\right)
$$

Performing the change of variables (4.11) in (4.17), we get

$$
\frac{1}{\varepsilon^{n}} \int_{B_{\frac{3}{4} N r(\varepsilon)}(\varepsilon \mathbf{i})} A\left(\frac{x}{\varepsilon}\right) D \zeta_{i}^{\varepsilon}\left(\frac{x-\varepsilon \mathbf{i}}{r(\varepsilon)}\right) D \zeta_{i}^{\varepsilon}\left(\frac{x-\varepsilon \mathbf{i}}{r(\varepsilon)}\right) \mathrm{d} x \leq \mu_{0, \varepsilon}^{N}(\varepsilon \mathbf{i})+\varepsilon .
$$

So

$$
\int_{F^{\varepsilon}} A\left(\frac{x}{\varepsilon}\right) D\left[\overline{v_{\varepsilon \mathbf{i}}^{-}} \zeta_{i}^{\varepsilon}\left(\frac{x-\varepsilon \mathbf{i}}{r(\varepsilon)}\right)\right] D\left[\overline{v_{\varepsilon \mathbf{i}}^{-}} \zeta_{i}^{\varepsilon}\left(\frac{x-\varepsilon \mathbf{i}}{r(\varepsilon)}\right)\right] \mathrm{d} x \leq \sum_{\mathbf{i}} \varepsilon^{n}\left|\overline{v_{\varepsilon \mathbf{i}}^{-}}\right|^{2} \mu_{0, \varepsilon}^{N}(\varepsilon \mathbf{i})+\varepsilon C .
$$

Hence, by (4.20)

$$
\begin{aligned}
\int_{\Omega} A^{\varepsilon} D \widetilde{v^{\varepsilon}} D \widetilde{v^{\varepsilon}} \mathrm{d} x & =\int_{\Omega \backslash F^{\varepsilon}} A^{\varepsilon} D v^{\varepsilon} D v^{\varepsilon} \mathrm{d} x+\int_{F^{\varepsilon}} A^{\varepsilon} D\left(\overline{v_{\varepsilon \mathbf{i}}^{+}}-\overline{v_{\varepsilon \mathbf{i}}^{-}} \zeta_{\mathbf{i}}^{\varepsilon}\left(\frac{x-\varepsilon \mathbf{i}}{r(\varepsilon)}\right)\right) D\left(\overline{v_{\varepsilon \mathbf{i}}^{+}}-\overline{v_{\varepsilon \mathbf{i}}^{-}} \zeta_{\mathbf{i}}^{\varepsilon}\left(\frac{x-\varepsilon \mathbf{i}}{r(\varepsilon)}\right)\right) \mathrm{d} x \\
& \leq \int_{\Omega} A^{\varepsilon} D v^{\varepsilon} D v^{\varepsilon} \mathrm{d} x+\sum_{\mathbf{i}} \varepsilon^{n}\left|\overline{v_{\varepsilon \mathbf{i}}^{-}}\right|^{2} \mu_{0, \varepsilon}^{N}(\varepsilon \mathbf{i})+\varepsilon C .
\end{aligned}
$$

Putting

$$
\bar{\mu}_{0, \varepsilon}^{N}=\sum_{\mathbf{i} \in \mathbb{Z}^{\varepsilon}} \mu_{0, \varepsilon}^{N}(\varepsilon \mathbf{i}) \chi_{Y_{\varepsilon \mathbf{i}}}
$$

and again

$$
g^{\varepsilon}=\sum_{\mathbf{i} \in \mathbb{Z}^{\varepsilon}}\left|\overline{v_{\varepsilon \dot{\mathbf{i}}}^{-}}\right|^{2} \chi_{Y_{\varepsilon \mathbf{i}}}
$$

by Lemma 4.5 , we have

$$
\begin{aligned}
\limsup _{\varepsilon \rightarrow 0} \int_{F^{\varepsilon}} A\left(\frac{x}{\varepsilon}\right) D \widetilde{v^{\varepsilon}} D \widetilde{v^{\varepsilon}} \mathrm{d} x & \leq \limsup _{\varepsilon \rightarrow 0} \sum_{\mathbf{i}} \varepsilon^{n}\left|\overline{v_{\varepsilon \mathbf{i}}^{-}}\right|^{2} \mu_{0, \varepsilon}^{N}(\varepsilon \mathbf{i}) \\
& =\limsup _{\varepsilon \rightarrow 0} \int_{\Omega} \bar{\mu}_{0, \varepsilon}^{N} g^{\varepsilon} \mathrm{d} x=\int_{\Omega} \mu_{0}^{N}\left(v^{-}\right)^{2} \mathrm{~d} x
\end{aligned}
$$

Since the linear term in the functional $J^{\varepsilon}$ passes immediately to the limit, as $\varepsilon \rightarrow 0$, letting $N \rightarrow \infty$, from (4.19)-(4.22) we get immediately

$$
\limsup _{\varepsilon \rightarrow 0} J^{\varepsilon}\left(u^{\varepsilon}\right) \leq J^{0}(v)
$$

for any $v \in H_{0}^{1}(\Omega) \cap L^{\infty}(\Omega)$. Recalling (4.6) and (4.23), we have just proved that $u \in H_{0}^{1}(\Omega)$ satisfies

$$
\int_{\Omega} A^{0} D u D u \mathrm{~d} x+\left\langle\mu_{0},\left(u^{-}\right)^{2}\right\rangle-2 \int_{\Omega} f u \mathrm{~d} x \leq \int_{\Omega} A^{0} D v D v \mathrm{~d} x+\left\langle\mu_{0},\left(v^{-}\right)^{2}\right\rangle-2 \int_{\Omega} f v \mathrm{~d} x,
$$


for any $v \in H_{0}^{1}(\Omega) \cap L^{\infty}(\Omega)$, and hence, by density, for any $v \in H_{0}^{1}(\Omega)$. So the function $u$ is the unique solution of the minimization problem

$$
\left\{\begin{array}{l}
\text { Find } u \in H_{0}^{1}(\Omega) \text { such that } \\
J^{0}(u)=\inf _{v \in H_{0}^{1}(\Omega)} J^{0}(v) .
\end{array}\right.
$$

As $u$ is uniquely determined, the whole sequence $\widehat{u^{\varepsilon}}$ converges to $u$ and Theorem 4.1 is proved.

Proof of Lemma 4.5. Let $g^{\varepsilon}=\sum_{\mathbf{i} \in \mathbb{Z}^{\varepsilon}}\left|\overline{u_{\varepsilon \mathbf{i}}^{-}}\right|^{2} \chi_{Y_{\varepsilon \mathbf{i}}}$.

We know that $\left(\widehat{u^{\varepsilon}}\right)^{-} \rightarrow u^{-}$strongly in $L^{2}(\Omega)$. Also

$$
\limsup _{\varepsilon \rightarrow 0} \int_{\Omega} g^{\varepsilon} \mathrm{d} x \leq C
$$

Indeed, using Poincaré's inequality

$$
\left.\sum_{\mathbf{i} \in \mathbb{Z}^{\varepsilon}} \int_{Y_{\varepsilon \mathbf{i}}} \mid \widehat{u^{\varepsilon}}\right)^{-}-\left.\overline{u_{\varepsilon \mathbf{i}}^{-}}\right|^{2} \mathrm{~d} x \leq C \varepsilon^{2} \int_{\Omega}\left|D\left(\widehat{u^{\varepsilon}}\right)^{-}\right|^{2} \mathrm{~d} x
$$

we get

$$
\begin{aligned}
\limsup _{\varepsilon \rightarrow 0} \int_{\Omega} g^{\varepsilon} \mathrm{d} x & \left.\leq\left.\limsup _{\varepsilon \rightarrow 0} 2\left(\sum_{\mathbf{i} \in \mathbb{Z}^{\varepsilon}} \int_{Y_{\varepsilon \mathbf{i}}}\left|\left(\widehat{u^{\varepsilon}}\right)^{-}-\widehat{u_{\varepsilon \mathbf{i}}^{-}}\right|^{2}+\int_{\Omega} \mid \widehat{u^{\varepsilon}}\right)^{-}\right|^{2} \mathrm{~d} x\right) \\
& \leq 2 \int_{\Omega}\left|u^{-}\right|^{2} \mathrm{~d} x \leq C .
\end{aligned}
$$

So

$$
\begin{aligned}
I=\left.\int_{\Omega}\left|g^{\varepsilon}-\right| u^{-}\right|^{2} \mid \mathrm{d} x & =\left.\int_{\Omega}\left|g^{\varepsilon}-\right|\left(\widehat{u^{\varepsilon}}\right)^{-}\right|^{2}+\left|\left(\widehat{u^{\varepsilon}}\right)^{-}\right|^{2}-\left|u^{-}\right|^{2} \mid \mathrm{d} x \\
& \leq\left.\int_{\Omega}\left|g^{\varepsilon}-\right|\left(\widehat{u^{\varepsilon}}\right)^{-}\right|^{2}\left|\mathrm{~d} x+\int_{\Omega}\right|\left|\left(\widehat{u^{\varepsilon}}\right)^{-}\right|^{2}-\left|u^{-}\right|^{2} \mid \mathrm{d} x .
\end{aligned}
$$

It suffices to evaluate the limsup of the first term of the right-hand side of (4.24).

$$
\begin{aligned}
\left.\limsup _{\varepsilon \rightarrow 0} \int_{\Omega}\left|g^{\varepsilon}-\right| \widehat{u^{\varepsilon}}\right)\left.^{-}\right|^{2} \mid \mathrm{d} x & \left.\leq\left. C \limsup _{\varepsilon \rightarrow 0} \sum_{\mathbf{i} \in \mathbb{Z}^{\varepsilon}} \int_{Y_{\varepsilon \mathbf{i}}}|| \overline{u_{\varepsilon \mathbf{i}}^{-}}\right|^{2}-\mid \widehat{u^{\varepsilon}}\right)\left.^{-}\right|^{2} \mid \mathrm{d} x \\
& \leq C \limsup _{\varepsilon \rightarrow 0} \sum_{\mathbf{i} \in \mathbb{Z}^{\varepsilon}} \int_{Y_{\varepsilon \mathbf{i}}}\left|\overline{u_{\varepsilon \mathbf{i}}^{-}}-\left(\widehat{u^{\varepsilon}}\right)^{-}\right|\left|\overline{u_{\varepsilon \mathbf{i}}^{-}}+\left(\widehat{u^{\varepsilon}}\right)^{-}\right| \mathrm{d} x .
\end{aligned}
$$


By Hölder's inequality

$$
\begin{aligned}
\limsup _{\varepsilon \rightarrow 0} I & \left.\leq C \limsup _{\varepsilon \rightarrow 0}\left(\sum_{\mathbf{i} \in \mathbb{Z}^{\varepsilon}} \int_{Y_{\varepsilon \mathbf{i}}}\left|\overline{u_{\varepsilon \mathbf{i}}^{-}}-\left(\widehat{u^{\varepsilon}}\right)^{-}\right|^{2} \mathrm{~d} x\right)^{\frac{1}{2}} \cdot \limsup _{\varepsilon \rightarrow 0}\left(\left.\int_{\Omega}\left(g^{\varepsilon}+\mid \widehat{u^{\varepsilon}}\right)^{-}\right|^{2}\right) \mathrm{d} x\right)^{\frac{1}{2}} \\
& \leq C \lim _{\varepsilon \rightarrow 0} \varepsilon\left(\int_{\Omega}\left|D\left(\widehat{u^{\varepsilon}}\right)^{-}\right|^{2} \mathrm{~d} x\right)^{\frac{1}{2}}=0 .
\end{aligned}
$$

So

$$
\left.\limsup _{\varepsilon \rightarrow 0} \int_{\Omega}\left|g^{\varepsilon}-\right| u^{-}\right|^{2} \mid \mathrm{d} x=0
$$

and hence

$$
g^{\varepsilon} \rightarrow\left|u^{-}\right|^{2} \text { strongly in } L^{1}(\Omega)
$$

\subsection{Holes of the same size as the period}

As already mentioned in the introduction, the asymptotic behavior of the solution $u^{\varepsilon}$ in this case is governed by an obstacle problem associated to the homogenized medium, which we recall that it is represented by the classical homogenized matrix $A_{p}^{0}$ defined below.

Theorem 4.6. There exists an extension $\widehat{u^{\varepsilon}}$ of the solution $u^{\varepsilon}$ of the variational inequality (4.1), positive inside the holes, such that

$$
\widehat{u^{\varepsilon}} \rightarrow u \quad \text { weakly in } H_{0}^{1}(\Omega)
$$

where $u$ is the unique solution of

$$
\left\{\begin{array}{l}
u \in H_{0}^{1}(\Omega), u \geq 0 \quad \text { in } \Omega, \\
\int_{\Omega} A_{p}^{0} D u D u \mathrm{~d} x-2 \int_{\Omega} f u \mathrm{~d} x \leq \int_{\Omega} A_{p}^{0} D v D v \mathrm{~d} x-2 \int_{\Omega} f v \mathrm{~d} x \quad \forall v \in H_{0}^{1}(\Omega), v \geq 0 \quad \text { in } \Omega .
\end{array}\right.
$$

Here, $A_{p}^{0}=\left(a_{i j}^{0}\right)$ is the classical homogenized matrix, whose entries are defined as follows:

$$
a_{i j}^{0}=\frac{1}{\left|Y^{*}\right|} \int_{Y^{*}}\left(a_{i j}(y)+a_{i k}(y) \frac{\partial \chi_{j}}{\partial y_{k}}\right) \mathrm{d} y
$$

in terms of the functions $\chi_{j}, j=1, \ldots, n$, solutions of the so-called cell problems

$$
\left\{\begin{array}{l}
-\operatorname{div}_{y} A(y)\left(D_{y} \chi_{j}+\mathbf{e}_{j}\right)=0 \text { in } Y^{*}, \\
A(y)\left(D \chi_{j}+\mathbf{e}_{j}\right) \cdot \nu=0 \text { on } \partial T \\
\chi_{j} \in H_{\# Y}^{1}\left(Y^{\star}\right), \int_{Y^{\star}} \chi_{j}=0
\end{array}\right.
$$

where $\mathbf{e}_{i}, 1 \leq i \leq n$, are the elements of the canonical basis in $\mathbb{R}^{n}$. The constant matrix $A^{0}$ is symmetric and positive-definite. 
Proof. Let $u^{\varepsilon}$ be the solution of the variational inequality (4.1). We shall use the same extension $\widehat{u^{\varepsilon}}$ as in the previous cases (see (2.12)). It is not difficult to see that $\widehat{u^{\varepsilon}}$ is bounded in $H_{0}^{1}(\Omega)$. So by extracting a subsequence, one has

$$
\widehat{u^{\varepsilon}} \rightarrow u \quad \text { weakly in } H_{0}^{1}(\Omega) \text {. }
$$

Since $u^{\varepsilon} \in K^{\varepsilon}$, then $\widehat{u^{\varepsilon}} \geq 0$ on the holes, i.e. $\widehat{u^{\varepsilon}} \chi_{T^{\varepsilon}} \geq 0$ in $\Omega$. Passing to the limit, we get $u(1-\theta) \geq 0$, $0<\theta<1$ in $\Omega$, and therefore $u \geq 0$ in $\Omega$.

Let $\varphi \in \mathcal{D}(\Omega), \varphi \geq 0$ and $\psi \in \mathcal{D}(\Omega), 0 \leq \psi \leq 1$ with $\psi \equiv 1$ on $\operatorname{supp}(\varphi)$. By classical regularity results $\chi_{i} \in L^{\infty}$. Using the boundedness of $\chi_{i}$ and $\varphi$, there exists $M \geq 0$ such that

$$
\left\|\frac{\partial \varphi}{\partial x_{i}}\right\|_{L^{\infty}}\left\|\chi_{i}\right\|_{L^{\infty}}<M
$$

Let

where $\delta$ is chosen such that

$$
v^{\varepsilon}=\left(\varphi+\sum_{i} \varepsilon \frac{\partial \varphi}{\partial x_{i}}(x) \chi_{i}\left(\frac{x}{\varepsilon}\right)+\delta\right) \psi
$$

$$
\varepsilon\left\|\frac{\partial \varphi}{\partial x_{i}}\right\|_{L^{\infty}}\left\|\chi_{i}\right\|_{L^{\infty}}<M \varepsilon=\delta .
$$

Then $v^{\varepsilon} \geq 0$ in $\Omega$ if $\varepsilon$ is enough small. Also $v^{\varepsilon} \in H_{0}^{1}(\Omega)$, and hence $v^{\varepsilon} \in K^{\varepsilon}$ which will allow us to take it as a test function in (4.1). Moreover, $v^{\varepsilon} \rightarrow \varphi$ strongly in $L^{2}(\Omega)$.

Let us compute $D v^{\varepsilon}$ :

$$
D v^{\varepsilon}=D \varphi+\sum_{i} \frac{\partial \varphi}{\partial x_{i}}(x) D \chi_{i}\left(\frac{x}{\varepsilon}\right)+\varepsilon\left(\sum_{i} D \frac{\partial \varphi}{\partial x_{i}}(x) \chi_{i}\left(\frac{x}{\varepsilon}\right)+M D \psi\right) .
$$

So

$$
D v^{\varepsilon}=\sum_{i} \frac{\partial \varphi}{\partial x_{i}}(x)\left(\mathbf{e}_{i}+D \chi_{i}\left(\frac{x}{\varepsilon}\right)\right)+\varepsilon\left(\sum_{i} D \frac{\partial \varphi}{\partial x_{i}}(x) \chi_{i}\left(\frac{x}{\varepsilon}\right)+M D \psi\right) .
$$

Using $v^{\varepsilon}$ as a test function in (4.1), we can write

$$
\int_{\Omega^{\varepsilon}} A^{\varepsilon} D u^{\varepsilon} D v^{\varepsilon} \mathrm{d} x \geq \int_{\Omega^{\varepsilon}} f\left(v^{\varepsilon}-u^{\varepsilon}\right) \mathrm{d} x+\int_{\Omega^{\varepsilon}} A^{\varepsilon} D u^{\varepsilon} D u^{\varepsilon} \mathrm{d} x .
$$

In fact, we have

$$
\int_{\Omega} D \widehat{u^{\varepsilon}}\left(\widetilde{A^{\varepsilon} D v^{\varepsilon}}\right) \mathrm{d} x \geq \int_{\Omega^{\varepsilon}} f\left(v^{\varepsilon}-u^{\varepsilon}\right) \mathrm{d} x+\int_{\Omega^{\varepsilon}} A^{\varepsilon} D u^{\varepsilon} D u^{\varepsilon} \mathrm{d} x .
$$

Denote

$$
\theta A_{p}^{0} \mathbf{e}_{j}=\frac{1}{\left|Y^{*}\right|} \int_{Y^{*}}{ }^{t} A(y)\left(D \chi_{j}+\mathbf{e}_{j}\right) \mathrm{d} y
$$

where $\theta=\left|Y^{*}\right| /|Y|$. Neglecting the term $\varepsilon\left(\sum_{i} D \frac{\partial \varphi}{\partial x_{i}}(x) \chi_{i}\left(\frac{x}{\varepsilon}\right)+M D \psi\right)$ which actually tends strongly to zero, we can pass immediately to the limit in the left-hand side of (4.28). Hence

$$
\int_{\Omega} D \widehat{D u^{\varepsilon}}\left(\widetilde{A^{\varepsilon} D v^{\varepsilon}}\right) \mathrm{d} x \rightarrow \int_{\Omega} \theta A_{p}^{0} D u D \varphi \mathrm{d} x .
$$


It is not difficult to pass to the limit in the first term of the right-hand side of (4.28). Indeed, since $v^{\varepsilon} \rightarrow \varphi$ strongly in $L^{2}(\Omega)$, we get

$$
\int_{\Omega^{\varepsilon}} f\left(v^{\varepsilon}-u^{\varepsilon}\right) \mathrm{d} x=\int_{\Omega} f \chi_{\Omega^{\varepsilon}}\left(v^{\varepsilon}-\widehat{u^{\varepsilon}}\right) \mathrm{d} x \rightarrow \int_{\Omega} f \theta(\varphi-u) \mathrm{d} x .
$$

So, it remains to pass to the limit only in the last term of (4.28). For doing this, we can write down the subdifferential inequality

$$
\int_{\Omega^{\varepsilon}} A^{\varepsilon} D u^{\varepsilon} D u^{\varepsilon} \mathrm{d} x \geq \int_{\Omega^{\varepsilon}} A^{\varepsilon} D w^{\varepsilon} D w^{\varepsilon} \mathrm{d} x+2 \int_{\Omega^{\varepsilon}} A^{\varepsilon} D w^{\varepsilon}\left(D u^{\varepsilon}-D w^{\varepsilon}\right) \mathrm{d} x,
$$

for any $w^{\varepsilon} \in H_{0}^{1}(\Omega), w^{\varepsilon} \geq 0$ in $\Omega$. Reasoning as before and choosing

$$
w^{\varepsilon}=\left(\bar{\varphi}+\sum_{i} \varepsilon \frac{\partial \bar{\varphi}}{\partial x_{i}}(x) \chi_{i}\left(\frac{x}{\varepsilon}\right)+\varepsilon \bar{M}\right) \bar{\psi}
$$

where $\bar{\varphi}, \bar{\psi}$ and $\bar{M}$ enjoy similar properties as the corresponding $\varphi, \psi$ and $M$, the right-hand side of the inequality (4.32) passes to the limit and one has

$$
\liminf _{\varepsilon \rightarrow 0} \int_{\Omega^{\varepsilon}} A^{\varepsilon} D u^{\varepsilon} D u^{\varepsilon} \mathrm{d} x \geq \int_{\Omega} \theta A_{p}^{0} D \bar{\varphi} D \bar{\varphi} \mathrm{d} x+2 \int_{\Omega} \theta A_{p}^{0} D \bar{\varphi}(D u-D \bar{\varphi}) \mathrm{d} x,
$$

for any $\bar{\varphi} \in \mathcal{D}(\Omega), \bar{\varphi} \geq 0$. But since $u \in H_{0}^{1}(\Omega)$ and $u \geq 0$ in $\Omega$, taking $\bar{\varphi} \rightarrow u$ strongly in $H_{0}^{1}(\Omega)$, we conclude

$$
\liminf _{\varepsilon \rightarrow 0} \int_{\Omega^{\varepsilon}} A^{\varepsilon} D u^{\varepsilon} D u^{\varepsilon} \mathrm{d} x \geq \int_{\Omega} \theta A_{p}^{0} D u D u \mathrm{~d} x .
$$

Putting together (4.30), (4.31) and (4.33), we get

$$
\int_{\Omega} \theta A_{p}^{0} D u D \varphi \mathrm{d} x \geq \int_{\Omega} f \theta(\varphi-u) \mathrm{d} x+\int_{\Omega} \theta A_{p}^{0} D u D u \mathrm{~d} x,
$$

for any $\varphi \in \mathcal{D}(\Omega), \varphi \geq 0$, and hence by density for any $v \in H_{0}^{1}(\Omega), v \geq 0$.

So, finally, we obtain

$$
\int_{\Omega} A_{p}^{0} D u D(v-u) \mathrm{d} x \geq \int_{\Omega} f(v-u) \mathrm{d} x
$$

which is just the limit problem (4.26). This completes the proof of Theorem 4.6.

Remark 4.7. The choice of the test function (4.27) gives, in fact, a first-corrector term for the weak convergence of $\widehat{u^{\varepsilon}}$ to $u$.

Acknowledgements. This work has been partially supported by Fondap through its Programme on Mathematical Mechanics. The authors gratefully acknowledge the support of the Chilean and French Governments through the Scientific Committee Ecos-Conicyt. The third author wishes to thank the Centro de Modelamiento Matemático de la Universidad de Chile for its warm hospitality and support. 


\section{REFERENCES}

[1] N. Ansini and A. Braides, Asymptotic analysis of periodically perforated non-linear media. J. Math. Pures Appl. 81 (2002) 439-451.

[2] H. Attouch, Variational Convergence for Functions and Operators. Pitman, London (1984).

[3] A. Braides and A. Defranceschi, Homogenization of Multiple Integrals. Oxford University Press, Oxford (1998).

[4] H. Brézis, Problèmes unilatéraux. J. Math. Pures Appl. 51 (1972) 1-168.

[5] C. Calvo Jurado and J. Casado Díaz, The limit of Dirichlet systems for variable monotone operators in general perforated domains. J. Math. Pures Appl. 81 (2002) 471-493.

[6] J. Casado Díaz, Existence of a sequence satisfying Cioranescu-Murat conditions in homogenization of Dirichlet problems in perforated domains. Rend. Mat. Appl. 16 (1996) 387-413.

[7] J. Casado-Díaz, Homogenization of Dirichlet problems for monotone operators in varying domains. Proc. Roy. Soc. Edinburgh Sect. A 127 (1997) 457-478.

[8] D. Cioranescu and J. Saint Jean Paulin, Homogenization in open sets with holes. J. Math. Anal. Appl. 71 (1979) $590-607$.

[9] D. Cioranescu and F. Murat, Un terme étrange venu d'ailleurs, I and II, in Nonlinear Differential Equations and Their Applications, Collège de France Seminar, H. Brézis and J.L. Lions, Eds., Vol. II, pp. 98-138, Vol. III, pp. 154-178. Pitman, London, Research Notes in Mathematics 60 and 70 (1982 and 1983). English translation: A Strange Term Coming from Nowhere, in Topics in the Mathematical Modelling of Composite Materials, A. Cherkaev and R.V. Kohn, Eds. Birkhäuser, Boston, Progr. Nonlinear Differential Equations Appl. 31 (1997) 45-93.

[10] C. Conca and P. Donato, Non homogeneous Neumann problems in domains with small holes. RAIRO Modél. Math. Anal. Numér. 22 (1988) 561-607.

[11] C. Conca and M. Vanninathan, On uniform $H^{2}$-estimates in periodic homogenization. Proc. Roy. Soc. Edinburgh A 131 (2001) 499-517.

[12] G. Dal Maso, On the integral representation of certain local functionals. Ricerche Mat. 32 (1983) 85-113.

[13] G. Dal Maso, Г-convergence and $\mu$-capacities. Ann. Scuola Norm. Sup. Pisa Cl. Sci. 14 (1987) 423-464.

[14] G. Dal Maso, An Introduction to $\Gamma-$ Convergence. Birkhäuser, Boston, Progr. Nonlinear Differential Equations Appl. 8 (1993).

[15] G. Dal Maso and A. Garroni, New results on the asymptotic behavior of Dirichlet problems in perforated domains. Math. Models Methods Appl. Sci. 4 (1994) 373-407.

[16] G. Dal Maso and F. Murat, Asymptotic behaviour and correctors for Dirichlet problems in perforated domains with homogeneous monotone operators. Ann. Scuola. Norm. Sup. Pisa Cl. Sci. 24 (1997) 239-290.

[17] G. Dal Maso and F. Murat, Asymptotic behaviour and correctors for linear Dirichlet problems with simultaneously varying operators and domains. Ann. Inst. H. Poincaré, Anal. non linéaire (to appear).

[18] E. De Giorgi and T. Franzoni, Su un tipo di convergenza variazionale. Atti. Accad. Naz. Lincei Rend. Cl. Sci. Mat. Fis. Natur. 58 (1975) 842-850.

[19] G. Duvaut and J.L. Lions, Les inéquations en mécanique et en physique. Dunod, Paris (1972).

[20] G. Fichera, Problemi elastostatici con vincoli unilaterali: il problema di Signorini con ambigue condizioni al contorno. Mem. Accad. Naz. Lincei, Serie 87 (1964) 91-140.

[21] A. Kovalevsky, An effect of double homogenization for Dirichlet problems in variable domains of general structure. C. R. Acad. Sci. Paris Sér. I Math. 328 (1999) 1151-1156.

[22] J.L. Lions and G. Stampacchia, Variational inequalities. Comm. Pure Appl. Math. 20 (1967) 493-519.

[23] V.A. Marcenko and E.Ja. Hrouslov, Boundary Value Problems in Domains with Fine-Grained Boundary (in russian). Naukova Dumka, Kiev (1974).

[24] F. Murat, H-convergence, Séminaire d'Analyse Fonctionnelle et Numérique de l'université d'Alger 1977-1978, multigraphied, 34 p. English translation: F. Murat and L. Tartar, H-convergence, in Topics in the Mathematical Modelling of Composite Materials, A. Cherkaev and R.V. Khon, Eds. Birkhäuser, Boston, Progr. Nonlinear Differential Equations Appl. 31 (1997) $21-43$.

[25] A. Signorini, Sopra alcune questioni di Elastostatica. Atti della Soc. Ital. per il Progresso della Scienze (1963).

[26] I.V. Skrypnik, Nonlinear Elliptic Boundary Value Problems. Teubner-Verlag, Leipzig (1986).

[27] L. Tartar, Problèmes d’homogénéisation dans les équations aux dérivées partielles. Cours Peccot, Collège de France (1977) (Partially written in [24]).

[28] L. Tartar, Quelques remarques sur l'homogénéisation, in Functional Analysis and Numerical Analysis, Proceedings of the Japan-France Seminar 1976, H. Fujita, Ed. Japan Society for the Promotion of Science, Tokyo (1978) 469-482. 